\title{
A COMBINATORIAL REALIZATION OF KIRILLOV-RESHETIKHIN CRYSTALS FOR TYPE E ARISING FROM TRANSLATIONS
}

\author{
IL-SEUNG JANG
}

\begin{abstract}
The main purpose of this paper is to give a combinatorial realization of Kirillov-Reshetikhin (KR simply) crystals $B^{r, s}$ for type $\mathrm{E}_{n}^{(1)}$ with a minuscule node $r$ and $s \geqslant 1$. To do this, we describe explicitly the crystal of the quantum nilpotent subalgebra associated with the translation by the negative of the $r$-th fundamental weight. Then the crystal can be extended as an affine crystal, in which a certain subcrystal characterized by the $\varepsilon_{r}^{*}$-statistic is isomorphic to $B^{r, s}$ as an affine crystal, where $\varepsilon_{r}^{*}$ is also realized precisely in terms of triple and quadruple paths.
\end{abstract}

\section{Contents}

1. Introduction ........................ 1 . .

2. Preliminaries .................... 4

3. Simply braided reduced expressions . . . . . . . . . . . . 6

4. Crystals of the quantum nilpotent subalgebras of type E . . . 13

5. Kirillov-Reshetikhin crystals of type $E$ with minuscule nodes 18

6. Combinatorial description of the $\varepsilon_{r}^{*}$-statistic . . . . . . . . . 20

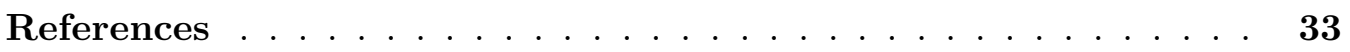

\section{INTRODUCTION}

Let $\mathfrak{g}$ be a finite-dimensional simple Lie algebra over $\mathbb{C}$. Let $\hat{\mathfrak{g}}$ be an affine Lie algebra corresponding to $\mathfrak{g}$ of untwisted type and let $U_{q}^{\prime}(\hat{\mathfrak{g}})$ be the quantum affine algebra associated to $\hat{\mathfrak{g}}$ without the derivation. The finite-dimensional irreducible $U_{q}^{\prime}(\hat{\mathfrak{g}})$-modules (of type 1) are classified by the Drinfeld polynomials [7] and it is wellknown that a certain class of such modules known as Kirillov-Reshetikhin modules

2010 Mathematics Subject Classification. 17B37, 22E46, 05E10.

Key words and phrases. quantum groups, affine crystals, Kirillov-Reshetikhin crystals, quantum nilpotent subalgebras, PBW crystals, type E.

This work is supported by the National Research Foundation of Korea(NRF) grant funded by the Korea government(MSIT) (No. 2020R1A5A1016126). 
plays an important role in the category of the finite-dimensional $U_{q}^{\prime}(\hat{\mathfrak{g}})$-modules (see, for example, [8]).

Let $W_{s, a}^{(r)}$ be the KR module associated to $1 \leqslant r \leqslant n, s \in \mathbb{Z}_{+}$and $a \in \mathbb{C}^{\times}$. It was conjectured by Hatayama et al. 12 , that for $1 \leqslant r \leqslant n$ and $s \in \mathbb{Z}_{+}$, there exists $a_{r, s} \in \mathbb{C}^{\times}$such that $W_{s, a_{r, s}}^{(r)}$ has the crystal pseudo-base introduced in [23] (cf. [20]). The conjecture have been proved for $r$ in the orbit of or adjacent to 0 in all affine types [22, 23], all non-exceptional types [38], types $\mathrm{G}_{2}^{(1)}$ and $\mathrm{D}_{4}^{(3)}$ [36], and types $\mathrm{E}_{6,7,8}^{(1)}, \mathrm{F}_{4}^{(1)}, \mathrm{E}_{6}^{(2)}$ with near adjoint nodes [37. Let $B^{r, s}$ be the crystal of the $\mathrm{KR}$ module, which is often called $K R$ crystal for short. Then it is an interesting problem to describe the structure of $B^{r, s}$ in a combinatorial way.

As the main result of this paper, we give a combinatorial realization of the KR crystal $B^{r, s}$ for type $\mathrm{E}_{n}^{(1)}$ with a minuscule node $r$ and $s \geqslant 1$ following the approach in $[16$.

Let us explain it in more details. Let $B(\infty)$ be the crystal associated to $U_{q}^{-}(\mathfrak{g})$, and let $B(\Lambda)$ be the crystal of an irreducible highest weight $U_{q}(\mathfrak{g})$-module with highest weight $\Lambda$, where $\Lambda$ is an integral dominant weight of $\mathfrak{g}$. It was known in [21] that there is a crystal embedding from $B(\Lambda)$ into $B(\infty)$, and the image of $B(\Lambda)$ is described by using the $\varepsilon_{i}^{*}$-statistic. Therefore, one can describe the crystal $B(\Lambda)$ in $B(\infty)$. By realizing $B(\infty)$ explicitly (see Section 4 ) in terms of $P B W$ basis [32, 39], we have a tensor product decomposition of $B(\infty)$, namely,

$$
B(\infty) \cong \mathbf{B}^{\mathrm{J}} \otimes \mathbf{B}_{\mathrm{J}}
$$

where $\mathbf{B}^{\mathrm{J}}$ and $\mathbf{B}_{\mathrm{J}}$ are subcrystals of $B(\infty)$ given in (4.3). By (1.1) and the characterization of $B\left(s \varpi_{r}\right)$ in $B(\infty)$, we also have

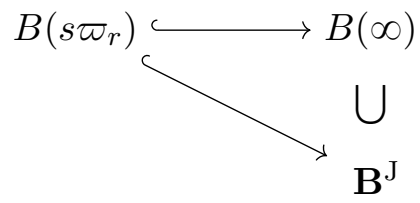

In other words, the crystal $\mathbf{B}^{\mathrm{J}}$ can be viewed as a limit of $B\left(s \varpi_{r}\right)$ as $s \rightarrow \infty$. Moreover, it can be extended as an affine crystal by giving 0-crystal operator explicitly (see Section 5). Then we denote by $\mathbf{B}^{\mathrm{J}, s}$ the image of $B\left(s \varpi_{r}\right)$ in $\mathbf{B}^{\mathrm{J}}$, which is an affine crystal. It was well-known in [6] that $B^{r, s} \cong B\left(s \varpi_{r}\right)$ classically, that is, by ignoring 0 -crystal operators, for a minuscule $r$. Then we will prove that

$$
\mathbf{B}^{\mathrm{J}, s} \cong B^{r, s},
$$

which is the main result of this paper. In the proof, the uniqueness of KR crystals for type E [18] (cf. Remark [5.2) plays a crucial role (see also 9] for non-exceptional types). 
We should remark that there are several realizations of $B(\infty)$. For example, a polyhedral realization of $B(\infty)$ was known by Nakashima-Zelevinsky in [35. It would be interesting to interpret $\mathbf{B}^{\mathrm{J}}$ and $\mathbf{B}^{\mathrm{J}, s}$ in this realization as affine crystals (cf. Remark 6.6).

The motivation of this work is an observation that a reduced expression $\mathbf{i}^{\mathrm{J}}$ obtained from $t_{-\varpi_{r}}$ is simply braided in the sense of [40] up to 2-term braid moves, which was essentially introduced by Littelmann [31] (cf. [40]). This yield a nice reduced expression of the longest element $w_{0}$ of Weyl group by which it enables us to describe the structure of $B(\infty)$ explicitly. In particular, the crystal operators of $B(\infty)$ are given as the signature rule induced from the tensor product rule of crystals, and we also obtain an explicit formula of the $\varepsilon_{r}^{*}$-statistic on $\mathbf{B}^{\mathrm{J}} \subset B(\infty)$ in terms of a combinatorial object in Definition 6.1, which is called path.

A combinatorial model of the $\mathrm{KR}$ crystals $B^{r, s}$ in terms of tableaux was already known in [18] for type $\mathrm{E}_{6}^{(1)}$ with $r=1,2,6$ and in [1] for type $\mathrm{E}_{7}^{(1)}$ with $r=7$. Both of them use a promotion operator to define the 0-crystal operators as in [41] for type $\mathrm{A}_{n}^{(1)}$ (cf. 9]). On the other hand, there exists another model of $B^{r, s}$ without the promotion operator for type $\mathrm{A}_{n}^{(1)}$ and types $\mathrm{D}_{n+1}^{(1)}, \mathrm{C}_{n}^{(1)}, \mathrm{D}_{n}^{(1)}$ with exceptional nodes $r$ [26] by using the RSK correspondence [25].

It would be interesting to ask whether there exists a tableau model of $B^{r, s}$ without a promotion operator for type $\mathrm{E}_{n}^{(1)}$ and then an isomorphism of affine crystals between our model and a (previous) tableau model in type $\mathrm{E}_{n}^{(1)}$. We would like to remark that it was proved in [26] that the RSK correspondence can be extended to the isomorphisms of affine crystals of type $\mathrm{A}_{n}^{(1)}$ between the tableau and polytope model of $B^{r, s}$. Recently, it is also shown in [16] that Burge correspondence [3], which is an analog of the RSK for type $\mathrm{D}_{n}$, can be extended to an isomorphism of affine crystals of type $\mathrm{D}_{n}^{(1)}$ between the tableau model of $B^{n, s}$ in [26] and the polytope model of $B^{n, s}$.

On the other hand, it is still an open problem to describe KR crystals for all types in a uniform way. There are several results by many authors related to this problem, see [28, 30, 29] and references therein. We hope to find a connection with the results to understand the structure of KR crystals more deeply and extend the approach of this paper beyond minuscule cases if it is possible.

This paper is organized as follows. In Section 2, we review the necessary background on the crystals. In Section 3 , we give the simply braided reduced expressions for types $\mathrm{ADE}$. In Section 4 , for type $\mathrm{E}_{n}$ with $n=6,7$, we describe the crystal operators of the crystal of $U_{q}^{-}(\mathfrak{g})$ and then we obtain the crystal $\mathbf{B}^{\mathrm{J}}$ of the quantum nilpotent subalgebra associated to $t_{-\varpi_{r}}$. In Section 5, we give a combinatorial realization of the KR crystal $B^{r, s}$ for type $\mathrm{E}_{n}^{(1)}$ with a minuscule $r$ (Theorem [5.1). In 
Section 6, we provide an explicit combinatorial formula of $\varepsilon_{r}^{*}$ on $\mathbf{B}^{\mathrm{J}}$ in terms of the triple and quadruple paths (Theorem 6.4).

Acknowledgement. The author is very grateful to Prof. Jae-Hoon Kwon for many stimulating, helpful discussions and encouraging him in the preparation of this paper; to Prof. Euiyong Park for his interest in this work and valuable comments. He would like to thank Prof. Cristian Lenart for letting him know the references [28, 29, 30] with his kind explanation and suggesting possible directions, and Travis Scrimshaw for his interest in this work, stimulating discussions, and letting him know the references [13, 42. Finally, he also would like to thank the anonymous referee for helpful and detailed comments.

\section{Preliminaries}

2.1. Crystals. Let us give a brief review on crystals (see [14, 20, 21] for more details). Let $\mathbb{Z}_{+}$denote the set of non-negative integers. Let $\mathfrak{g}$ be the Kac-Moody algebra associated to a symmetrizable generalized Cartan matrix $A=\left(a_{i j}\right)_{i, j \in \mathrm{I}}$ with an index set I. Let $P^{\vee}$ be the dual weight lattice, $P=\operatorname{Hom}_{\mathbb{Z}}\left(P^{\vee}, \mathbb{Z}\right)$ the weight lattice, $Q$ the root lattice, $\Pi^{\vee}=\left\{h_{i} \mid i \in I\right\} \subset P^{\vee}$ the set of simple coroots, and $\Pi=\left\{\alpha_{i} \mid i \in I\right\} \subset P$ the set of simple roots of $\mathfrak{g}$ such that $\left\langle\alpha_{j}, h_{i}\right\rangle=a_{i j}$ for $i, j \in \mathrm{I}$. Let $P^{+}$be the set of integral dominant weights and let $\varpi_{i}$ be the $i$ th fundamental weight for $i \in \mathrm{I}$. For an indeterminate $q$, let $U_{q}(\mathfrak{g})$ be the quantized enveloping algebra of $\mathfrak{g}$ generated by $e_{i}, f_{i}$, and $q^{h}$ for $i \in \mathrm{I}$ and $h \in P^{\vee}$ over $\mathbb{Q}(q)$. We denote by $U_{q}^{-}(\mathfrak{g})$ the subalgebra of $U_{q}(\mathfrak{g})$ generated by $f_{i}$ for all $i \in \mathrm{I}$.

A $\mathfrak{g}$-crystal (or crystal if there is no confusion on $\mathfrak{g}$ ) is a set $B$ together with the maps wt $: B \rightarrow P, \varepsilon_{i}, \varphi_{i}: B \rightarrow \mathbb{Z} \cup\{-\infty\}$ and $\tilde{e}_{i}, \tilde{f}_{i}: B \rightarrow B \cup\{\boldsymbol{0}\}$ for $i \in \mathrm{I}$ satisfying certain axioms. We denote by $B(\infty)$ the crystal associated to $U_{q}^{-}(\mathfrak{g})$. Let * be the $\mathbb{Q}(q)$-linear anti-automorphism of $U_{q}(\mathfrak{g})$ such that $e_{i}^{*}=e_{i}, f_{i}^{*}=f_{i}$, and $\left(q^{h}\right)^{*}=q^{-h}$ for $i \in \mathrm{I}$ and $h \in P$. Then $*$ induces a bijection on $B(\infty)$. For $i \in \mathrm{I}$, we define $\tilde{e}_{i}^{*}=* \circ \tilde{e}_{i} \circ *$ and $\tilde{f}_{i}^{*}=* \circ \tilde{f}_{i} \circ *$ on $B(\infty)$. In particular, $\varepsilon_{i}^{*}(b)$ is defined by $\max \left\{n \mid \tilde{e}_{i}^{* n} b \neq \mathbf{0}\right\}$. For $\Lambda \in P^{+}$, we denote by $B(\Lambda)$ the crystal associated to an irreducible highest weight $U_{q}(\mathfrak{g})$-module $V(\Lambda)$ with highest weight $\Lambda$. For $\mu \in P$, let $T_{\mu}=\left\{t_{\mu}\right\}$ be a crystal, where $\operatorname{wt}\left(t_{\mu}\right)=\mu$, and $\varphi_{i}\left(t_{\mu}\right)=-\infty$ for all $i \in \mathrm{I}$.

For crystals $B_{1}$ and $B_{2}$, the tensor product $B_{1} \otimes B_{2}$ is defined to be $B_{1} \times B_{2}$ as a set with elements denoted by $b_{1} \otimes b_{2}$, where

$$
\begin{gathered}
\operatorname{wt}\left(b_{1} \otimes b_{2}\right)=\operatorname{wt}\left(b_{1}\right)+\operatorname{wt}\left(b_{2}\right), \\
\varepsilon_{i}\left(b_{1} \otimes b_{2}\right)=\max \left\{\varepsilon_{i}\left(b_{1}\right), \varepsilon_{i}\left(b_{2}\right)-\left\langle\operatorname{wt}\left(b_{1}\right), h_{i}\right\rangle\right\}, \\
\varphi_{i}\left(b_{1} \otimes b_{2}\right)=\max \left\{\varphi_{i}\left(b_{1}\right)+\left\langle\operatorname{wt}\left(b_{2}\right), h_{i}\right\rangle, \varphi_{i}\left(b_{2}\right)\right\},
\end{gathered}
$$




$$
\begin{aligned}
& \tilde{e}_{i}\left(b_{1} \otimes b_{2}\right)= \begin{cases}\tilde{e}_{i} b_{1} \otimes b_{2} & \text { if } \varphi_{i}\left(b_{1}\right) \geqslant \varepsilon_{i}\left(b_{2}\right), \\
b_{1} \otimes \tilde{e}_{i} b_{2} & \text { if } \varphi_{i}\left(b_{1}\right)<\varepsilon_{i}\left(b_{2}\right),\end{cases} \\
& \tilde{f}_{i}\left(b_{1} \otimes b_{2}\right)= \begin{cases}\tilde{f}_{i} b_{1} \otimes b_{2} & \text { if } \varphi_{i}\left(b_{1}\right)>\epsilon_{i}\left(b_{2}\right), \\
b_{1} \otimes \tilde{f}_{i} b_{2} & \text { if } \varphi_{i}\left(b_{1}\right) \leqslant \epsilon_{i}\left(b_{2}\right),\end{cases}
\end{aligned}
$$

for $i \in \mathrm{I}$. Here, we assume that $\mathbf{0} \otimes b_{2}=b_{1} \otimes \mathbf{0}=\mathbf{0}$. Then $B_{1} \otimes B_{2}$ is a crystal.

For two crystals $B$ and $B^{\prime}$, an isomorphism of crystals between $B$ and $B^{\prime}$ is a bijection $\Psi: B \cup\{\mathbf{0}\} \rightarrow B^{\prime} \cup\{\mathbf{0}\}$ such that $\Psi(\mathbf{0})=\mathbf{0}, \varepsilon_{i}(\Psi(b))=\varepsilon_{i}(b), \varphi_{i}(\Psi(b))=$ $\varphi_{i}(b), \tilde{e}_{i} \Psi(b)=\Psi\left(\tilde{e}_{i} b\right)$ and $\tilde{f}_{i} \Psi(b)=\Psi\left(\tilde{f}_{i} b\right)$ for all $i \in \mathrm{I}$.

2.2. Quantum nilpotent subalgebra $U_{q}^{-}(w)$. Let $W$ be Weyl group of $\mathfrak{g}$ generated by the simple reflections $s_{i}(i \in \mathrm{I})$. For $w \in W$, let $R(w)$ be the set of reduced expressions of $w$, that is,

$$
R(w)=\left\{\mathbf{i}=\left(i_{1}, \ldots, i_{m}\right) \mid w=s_{i_{1}} \ldots s_{i_{m}} \text { and } m \text { is minimal }\right\} .
$$

Let $\Phi^{+}$be the set of positive roots of $\mathfrak{g}$. It is well-known that for $\mathbf{i}=\left(i_{1}, \ldots, i_{m}\right) \in$ $R(w)$

$$
\left\{\beta_{1}:=\alpha_{i_{1}}, \beta_{2}:=s_{i_{1}}\left(\alpha_{i_{2}}\right), \cdots, \beta_{m}:=s_{i_{1}} \ldots s_{i_{m-1}}\left(\alpha_{i_{m}}\right)\right\} \subset \Phi^{+}
$$

(see [43] and reference therein).

For each $i \in \mathrm{I}$, let $T_{i}$ be the $\mathbb{Q}(q)$-algebra automorphism of $U_{q}(\mathfrak{g})$, where $T_{i}$ is equal to $T_{i, 1}^{\prime \prime}$ in [34. For $w \in W$ and $\mathbf{i}=\left(i_{1}, \ldots, i_{m}\right) \in R(w)$, put

$$
f_{\beta_{k}}=T_{i_{1}} \ldots T_{i_{k-1}}\left(f_{i_{k}}\right) \quad(1 \leqslant k \leqslant m),
$$

and for $\mathbf{c}=\left(c_{\beta_{1}}, \ldots, c_{\beta_{m}}\right) \in \mathbb{Z}_{+}^{m}$

$$
b_{\mathbf{i}}(\mathbf{c})=f_{\beta_{1}}^{\left(c_{\beta_{1}}\right)} f_{\beta_{2}}^{\left(c_{\beta_{2}}\right)} \cdots f_{\beta_{m}}^{\left(c_{\beta_{m}}\right)},
$$

where $f_{\beta_{k}}^{\left(c_{\beta_{k}}\right)}$ is the divided power of $f_{\beta_{k}}$ for $1 \leqslant k \leqslant m$. Then we denote by $U_{q}^{-}(w)$ the $\mathbb{Q}(q)$-subspace of $U_{q}^{-}(\mathfrak{g})$ generated by $\left\{b_{\mathbf{i}}(\mathbf{c}) \mid \mathbf{c} \in \mathbb{Z}^{m}\right\}$. It is known in [34] that $U_{q}^{-}(w)$ does not depend on $\mathbf{i}$. In particular, if $\mathfrak{g}$ is of finite type and $w_{0}$ is the longest element of $W$, then $U_{q}^{-}\left(w_{0}\right)=U_{q}^{-}(\mathfrak{g})$.

The commutation relation on the root vectors $f_{\beta_{k}}(1 \leqslant k \leqslant m)$ is well-known as Levendorskii-Soibelman formula (e.g. see [24] and references therein) and this implies that the subspace $U_{q}^{-}(w)$ is the $\mathbb{Q}(q)$-subalgebra of $U_{q}^{-}(\mathfrak{g})$ generated by $\left\{f_{\beta_{k}} \mid 1 \leqslant k \leqslant m\right\}$, which is called quantum nilpotent subalgebra associated to $w$. 
2.3. $\mathbf{P B W}$ crystal $\mathbf{B}_{\mathbf{i}}$. Assume that $\mathfrak{g}$ is of finite type. Let us review the formulation of $B(\infty)$ for finite types by using Poincaré-Birkhoff-Witt type bases [32, 33, 39] (cf. [20]).

Let us take $w=w_{0}$ in (2.2). Then the set of positive roots associated to $w_{0}$ is equal to $\Phi^{+}$, and the set

$$
B_{\mathbf{i}}:=\left\{b_{\mathbf{i}}(\mathbf{c}) \mid \mathbf{c} \in \mathbb{Z}_{+}^{N}\right\}
$$

is a $\mathbb{Q}(q)$-basis of $U_{q}^{-}(\mathfrak{g})$, which is often called a $P B W$ basis associated to i. Let $\mathcal{A}_{0}$ be the subring of $\mathbb{Q}(q)$ consisting of rational functions regular at $q=0$. The $\mathcal{A}_{0^{-}}$ lattice of $U_{q}^{-}(\mathfrak{g})$ generated by $B_{\mathbf{i}}$ is independent of the choice of $\mathbf{i}$, which we denote by $L(\infty)$. Let $\pi: L(\infty) \longrightarrow L(\infty) / q L(\infty)$ be the canonical projection. Let $B(\infty)$ be the image of $B_{\mathbf{i}}$ under $\pi$. Then it is known in [33, 39] that the pair $(L(\infty), B(\infty))$ coincides with the Kashiwara's crystal base of $U_{q}^{-}(\mathfrak{g})$ 20]

We identify

$$
\mathbf{B}_{\mathbf{i}}:=\mathbb{Z}_{+}^{N}
$$

with the crystal $\pi\left(B_{\mathbf{i}}\right)(=B(\infty))$ under the map $\mathbf{c} \mapsto b_{\mathbf{i}}(\mathbf{c})$, and call $\mathbf{c} \in \mathbf{B}_{\mathbf{i}}$ an $\mathbf{i}$ Lusztig data associated to $\mathbf{i}$. Then $\mathbf{B}_{\mathbf{i}}$ is called the crystal of $\mathbf{i}$-Lusztig datum. We also call it $P B W$ crystal for short if there is no confusion for $\mathbf{i}$.

\section{SimPLY BRAIDED REDUCED EXPRESSIONS}

3.1. Simply braided words. Let us recall that a total order $<$ on the set $\Phi^{+}$of positive roots. Let us assume that $\mathfrak{g}$ is of simply-laced type for simplicity. A order $<$ is called convex if either $\gamma<\gamma^{\prime}<\gamma^{\prime \prime}$ or $\gamma^{\prime \prime}<\gamma^{\prime}<\gamma$ whenever $\gamma^{\prime}=\gamma+\gamma^{\prime \prime}$ for $\gamma, \gamma^{\prime}, \gamma^{\prime \prime} \in \Phi^{+}$. It is well-known that there exists a one-to-one correspondence between $R\left(w_{0}\right)$ and the set of convex orders on $\Phi^{+}$, where the convex order $\prec$ associated with $\mathbf{i}=\left(i_{1}, \ldots, i_{N}\right) \in R\left(w_{0}\right)$ is given by

$$
\beta_{1} \prec \beta_{2} \prec \ldots<\beta_{N},
$$

where $\beta_{k}$ is as in (2.2) [43].

There exists a reduced expression $\mathbf{i}^{\prime} \in R\left(w_{0}\right)$ obtained from $\mathbf{i}$ by a 3 -term braid move $\left(i_{k}, i_{k+1}, i_{k+2}\right) \rightarrow\left(i_{k+1}, i_{k}, i_{k+1}\right)$ with $i_{k}=i_{k+2}$ if and only if $\left\{\beta_{k}, \beta_{k+1}, \beta_{k+2}\right\}$ forms the positive roots of type $A_{2}$, where the corresponding convex order $<^{\prime}$ is given by replacing $\beta_{k}<\beta_{k+1}<\beta_{k+2}$ with $\beta_{k+2} \prec^{\prime} \beta_{k+1} \prec^{\prime} \beta_{k}$. Also there exists a reduced expression $\mathbf{i}^{\prime} \in R\left(w_{0}\right)$ obtained from $\mathbf{i}$ by a 2 -term braid move $\left(i_{k}, i_{k+1}\right) \rightarrow\left(i_{k+1}, i_{k}\right)$ if and only if $\beta_{k}$ and $\beta_{k+1}$ are of type $A_{1} \times A_{1}$, where the associated convex ordering $\prec^{\prime}$ is given by replacing $\beta_{k}<\beta_{k+1}$ with $\beta_{k+1} \prec^{\prime} \beta_{k}$.

Definition 3.1. ([40, Definition 4.1]) Let $i \in \mathrm{I}$ be given. Then $\mathbf{i} \in R\left(w_{0}\right)$ is simply braided for $i$ if one can obtain $\mathbf{i}^{\prime}=\left(i_{1}^{\prime}, \ldots, i_{N}^{\prime}\right) \in R\left(w_{0}\right)$ with $i_{1}^{\prime}=i$ by applying to $\mathbf{i}$ a sequence of braid moves consisting of either a 2 -term move or 3 -term braid move 
such that $\left(\gamma, \gamma^{\prime}, \gamma^{\prime \prime}\right) \rightarrow\left(\gamma^{\prime \prime}, \gamma^{\prime}, \gamma\right)$ with $\gamma^{\prime \prime}=\alpha_{i}$. If $\mathbf{i}$ is simply braided for all $i \in \mathrm{I}$, then we say that $\mathbf{i}$ is simply braided.

3.2. Reduced expressions of the longest element of types ADE. Let us briefly review some facts related to the (extended) affine Weyl groups (see [4, 15, 19] for more details).

Let $\hat{\mathfrak{g}}$ be the affine Kac-Moody algebra of symmetric affine type with an index set $\hat{I}=\{0,1, \ldots, n\}$, where $\mathfrak{g}$ is the underlying finite-dimensional simple Lie algebra. We denote by $\widehat{W}$ the affine Weyl group of $\hat{\mathfrak{g}}$, that is, the group generated by simple reflections $s_{i}(i \in \hat{\mathrm{I}})$. It is well-known that $\widehat{W}$ is isomorphic to the semidirect product $W \ltimes Q$ under the identification $s_{0} \leftrightarrow\left(s_{\theta}, \theta\right)$ and $s_{i} \leftrightarrow\left(s_{i}, 0\right)$.

The extended affine Weyl group $\widetilde{W}$ is given by the semidirect product $W \ltimes P$. Let $\mathcal{T}$ be the set of bijections $\tau: \hat{\mathrm{I}} \rightarrow \hat{\mathrm{I}}$ such that $a_{\tau(i) \tau(j)}=a_{i j}$ for all $i, j \in \hat{\mathrm{I}}$. Each element $\tau \in \mathcal{T}$ induces a unique automorphism $\psi_{\tau}$ of $\widehat{W}$ so that $\psi_{\tau}\left(s_{i}\right)=s_{\tau(i)}$ for $i \in \hat{\mathrm{I}}$. Then it is known that $\widehat{W}$ is a normal subgroup of $\widetilde{W}$ such that $\mathcal{T} \simeq \widetilde{W} / \widehat{W}$ and $\widetilde{W} \simeq \mathcal{T} \ltimes \widehat{W}$, where the action of $\tau \in \mathcal{T}$ in $\widetilde{W}$ is given by $\psi_{\tau}$. A reduced expression of $w \in \widetilde{W}$ is defined by $w=\tau s_{i_{1}} \cdots s_{i_{m}}$, where $\tau \in \mathcal{T}$ and $s_{i_{1}} \cdots s_{i_{m}}$ is a reduced expression of $\tau^{-1} w \in \widehat{W}$.

Let us fix $r \in \mathrm{I}$ and let $t_{\varpi_{r}}$ be the translation by the $r$-th fundamental weight $\varpi_{r}$ of $\mathfrak{g}$ [19]. One can take a diagram automorphism $\tau_{r} \in \mathcal{T}$ so that $\tau_{r}^{-1} t_{\varpi_{r}} \in \widehat{W}$. Let $w^{\mathrm{J}}$ be the minimal length coset representative associated to $\mathrm{J}=\mathrm{I} \backslash\{r\}$. Then it is well-known (e.g. see [4]) that $t_{-\varpi_{r}} \tau_{r}=w^{\mathrm{J}}$.

Now, we assume that $\varpi_{r}$ is minuscule, that is, the weyl group $W$ acts transitively on the weights of $V\left(\varpi_{r}\right)$. Note that the complete list of $r \in I$ such that $\varpi_{r}$ is minuscule is as follows (e.g. see [4, 5]):

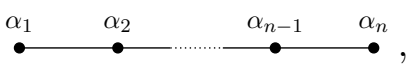

Type $\mathrm{A}_{n}$

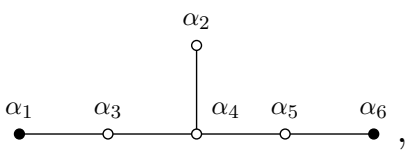

Type $\mathrm{E}_{6}$

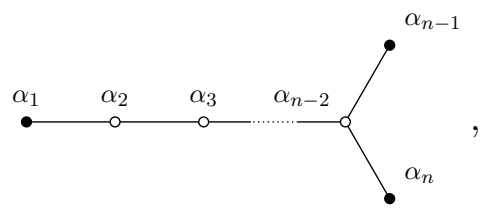

Type $\mathrm{D}_{n}$

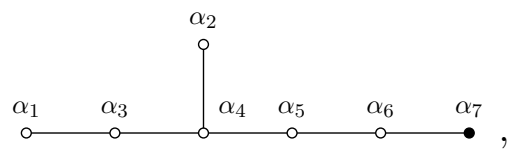

Type $\mathrm{E}_{7}$

Figure 1. The Dynkin diagrams for types $\mathrm{A}_{n}, \mathrm{D}_{n}, \mathrm{E}_{6}$ and $\mathrm{E}_{7}$ with minuscule nodes marked as 
The following reduced expressions of $w^{\mathrm{J}}$ for types ADE can be essentially found in [31, Sections 5, 7 and 8], respectively, where the enumeration of simple roots for types D and E in [31] are slightly different from the setting in this paper. We remark that the reduced expression in type A is slightly generalized form of the one in [31, Section 5].

Proposition 3.2. The reduced expression $\mathbf{i}^{\mathrm{J}}$ of $w^{\mathrm{J}}$ given as follows.

(1) for type $A_{n}$, we have

$$
\mathbf{i}^{\mathrm{J}}=\mathbf{i}_{1} \cdot \mathbf{i}_{2} \cdots \cdot \mathbf{i}_{n-r+1},
$$

where $\mathbf{i}_{s}=(r+s-1, r+s-2, \ldots, s+1, s)$ for $1 \leqslant s \leqslant n-r+1$.

(2) for type $\mathrm{D}_{n}$, we have

$$
\begin{cases}\mathbf{i}^{\mathrm{J}}=(1,2, \cdots, n-1, n, n-2, n-3, \cdots, 2,1) & \text { if } r=1, \\ \mathbf{i}^{\mathrm{J}}=\mathbf{i}_{1} \cdot \mathbf{i}_{2} \cdots \mathbf{i}_{n-1} & \text { if } r=n-1 \text { or } n,\end{cases}
$$

where $\mathbf{i}_{s}(1 \leqslant s \leqslant n-1)$ is given by

$$
\mathbf{i}_{s}= \begin{cases}(r, n-2, \ldots, k+1, k), & \text { if } s \text { is odd, } \\ (r-1, n-2, \ldots, k+1, k), & \text { if } s \text { is even and } r=n, \\ (r+1, n-2, \ldots, k+1, k), & \text { if } s \text { is even and } r=n-1, \\ (r), & \text { if } n \text { is even and } s=n-1 .\end{cases}
$$

(3) for type $\mathrm{E}_{6}$, we have

$$
\mathbf{i}^{\mathrm{J}}= \begin{cases}(1,3,4,5,6,2,4,5,3,4,2,1,3,4,5,6) & \text { if } r=1 \\ (6,5,4,3,1,2,4,3,5,4,2,6,5,4,3,1) & \text { if } r=6 .\end{cases}
$$

(4) for type $\mathrm{E}_{7}$, we have

$$
\mathbf{i}^{\mathrm{J}}=(7,6,5,4,3,1,2,4,3,5,4,2,6,5,4,3,1,7,6,5,4,3,2,4,5,6,7) .
$$

Let us take $w_{0}=w^{\mathrm{J}} w_{\mathrm{J}^{\star}}$ such that $w^{\mathrm{J}} w_{\mathrm{J}^{\star}}=w_{\mathrm{J}} w^{\mathrm{J}}$, where $w_{\mathrm{J}}$ is the longest element of the subgroup $W_{\mathrm{J}} \subset W$ generated by $s_{j}$ for $j \in \mathrm{J}$. Then we define the reduced expression $\mathbf{i}_{0} \in R\left(w_{0}\right)$ by

$$
\mathbf{i}_{0}=\mathbf{i}^{\mathrm{J}} \cdot \mathbf{i}_{\mathrm{J}^{\star}},
$$

where $\mathbf{i}^{\mathrm{J}}$ is given in Proposition 3.2 and $\mathbf{i}_{\mathrm{J}^{\star}}$ is a reduced expression of $w_{\mathrm{J}^{\star}}$. In particular, if $\mathfrak{g}$ is of types $\mathrm{E}_{6}$ or $\mathrm{E}_{7}$, then we may choose the reduced expression $\mathbf{i}_{\mathrm{J}^{\star}}$ 
by

$$
\mathbf{i}_{\mathrm{J}^{\star}}= \begin{cases}(5,4,3,1,2,4,3,5,4,2,5,4,3,1,5,4,3,5,4,5) & \text { for type } \mathrm{E}_{6} \text { with } r=1, \\ (3,4,5,6,2,4,5,3,4,2,3,4,5,6,3,4,5,3,4,3) & \text { for type } \mathrm{E}_{6} \text { with } r=6, \\ \mathbf{i}_{0}^{\mathrm{E}_{6}} & \text { for type } \mathrm{E}_{7} \text { with } r=7,\end{cases}
$$

where $\mathbf{i}_{0}^{\mathrm{E}_{6}}$ is equal to the reduced expression (3.2) of type $\mathrm{E}_{6}$ with $r=1$ or 6 . Note that the expression $\mathbf{i}_{\mathrm{J}^{\star}}$ for type $\mathrm{E}_{6}$ is obtained from (3.2) of type $\mathrm{D}_{5}$ associated to $\mathrm{J}$ (cf. [16, Section 3.1]).

The following property of $\mathbf{i}_{0}$ is a consequence of [40, 42]. For completeness, let us give an elementary proof of this property for type E.

Proposition 3.3. For $i \in \mathrm{I} \backslash\{r\}$, there exists a reduced expression $\mathbf{i} \in R\left(w_{0}\right)$, which is equal to $\mathbf{i}_{0}$ up to 2 -term braid moves, such that $\mathbf{i}$ is simply braided for $i$.

Proof. We state the proof for type $\mathrm{E}_{n}$, where the similar proof for other cases is given in [16].

Case 1. Type $\mathrm{E}_{6}$. Let us consider the case of $r=6$. Note that the proof for the case of $r=1$ is almost identical. Since $\mathbf{i}_{\mathrm{J}^{\star}}$ is the reduced expression of the longest element for type $\mathrm{D}_{5}$ associated to $\{2,3,4,5,6\}$ (cf. (4.2) ) by ignoring $\mathbf{i}^{\mathrm{J}}$, we know that it is simply braided for $k \neq 1$ [16, Proposition 3.2], that is, there exists $\mathbf{i}_{\mathrm{J}^{\star}}^{\prime}$ such that $k \cdot \mathbf{i}_{\mathrm{J}^{\star}}^{\prime}$ is obtained from $\mathbf{i}_{\mathrm{J}^{\star}}$ by 2 -term and 3 -term braid moves as in Definition 3.1. Then it is enough to check that $\mathbf{i}^{\mathrm{J}} \cdot k$ is simply braided for $k^{\star}$ up to 2-term braid moves, where $k^{\star}$ is given by

\begin{tabular}{|c||c|c|c|c|c|}
\hline$k$ & 2 & 3 & 4 & 5 & 6 \\
\hline$k^{*}$ & 5 & 2 & 4 & 3 & 1 \\
\hline
\end{tabular}

Let us consider

$$
\overline{\mathbf{i}}^{\mathrm{J}, k}= \begin{cases}(6,5,4,3,1,2,4,3,5, \mathbf{6}, \mathbf{4}, \mathbf{5}, \mathbf{2}, 4,3,1) & \text { if } k=2, \\ (6,5,4, \mathbf{2}, \mathbf{3}, \mathbf{4}, \mathbf{1}, 3,5,4,2,6,5,4,3,1) & \text { if } k=3, \\ (6,5,4,3,1,2,4, \mathbf{5}, \mathbf{3}, 4,2,6,5,4,3,1) & \text { if } k=4, \\ (6,5,4,3,1,2,4,3,5,4,2,6,5,4,3,1) & \text { if } k=5,6 .\end{cases}
$$

One can check that $\overline{\mathbf{i}}^{\mathrm{J}, k}$ is equal to $\mathbf{i}^{\mathrm{J}}$ up to 2-term braid moves which occur on bold numbers. Furthermore, it is straightforward to check that $\overline{\mathbf{i}}^{\mathrm{J}, k} \cdot k$ is simply braided for $k^{\star}$. Hence $\mathbf{i}:=\overline{\mathbf{i}}^{\mathrm{J}, k} \cdot \mathbf{i}_{\mathrm{J}}$ is simply braided for $k^{\star}$ and equal to $\mathbf{i}_{0}$ up to 2-term braid moves.

Case 2. Type $\mathrm{E}_{7}$. In this case, $\mathbf{i}_{\mathrm{J}^{\star}}$ is the reduced expression of the longest element for type $\mathrm{E}_{6}$ associated to $\{1,2,3,4,5,6\}$ (cf. (4.2)) by ignoring $\mathbf{i}^{\mathrm{J}}$, and it is simply 
braided for $k \neq 7$ since we take $\mathbf{i}_{J^{\star}}$ is equal to $\mathbf{i}_{0}$ for type $\mathrm{E}_{6}$ (3.3). Then, we apply the similar argument as in Case 1 by using

$$
\overline{\mathbf{i}}^{\mathrm{J}, k}= \begin{cases}(7,6,5,4,3,1,2,4,3,5,4,2, \mathbf{6 , 7}, \mathbf{5}, \mathbf{6}, \mathbf{4}, \mathbf{5}, \mathbf{3}, \mathbf{4}, \mathbf{1}, \mathbf{3}, 2,4,5,6,7) & \text { if } k=1, \\ (7,6,5,4, \mathbf{2}, \mathbf{3}, \mathbf{4}, \mathbf{1}, 3,5,4,2,6,5,4,3,1,7,6,5,4,3,2,4,5,6,7) & \text { if } k=2, \\ (7,6,5,4,3,1,2,4,3,5, \mathbf{6}, \mathbf{4}, \mathbf{5}, \mathbf{2}, 4,3,1,7,6,5,4, \mathbf{2}, \mathbf{3}, 4,5,6,7) & \text { if } k=3, \\ (7,6,5,4,3,1,2,4, \mathbf{5}, \mathbf{3}, 4,2,6,5,4,3,1,7,6,5,4,3,2,4,5,6,7) & \text { if } k=4, \\ (7,6,5,4,3,1,2,4,3,5,4,2,6,5,4,3,1,7,6,5,4,3,2,4,5,6,7) & \text { if } k=5,6 .\end{cases}
$$

Here $k^{\star}$ is given by

\begin{tabular}{|c||c|c|c|c|c|c|}
\hline$k$ & 1 & 2 & 3 & 4 & 5 & 6 \\
\hline$k^{*}$ & 6 & 2 & 5 & 4 & 3 & 1 \\
\hline
\end{tabular}

Then $\mathbf{i}:=\overline{\mathbf{i}}^{\mathrm{J}, k} \cdot \mathbf{i}_{\mathrm{J}^{\star}}$ is simply braided for $k^{\star}$ and equal to $\mathbf{i}_{0}$ up to 2 -term braid moves.

\section{Remark 3.4.}

(1) The reduced expression $\mathbf{i}^{\mathrm{J}}$ is unique up to 2-term braid moves in the sense of [17, Remark 5.2] (see also [31, 42]).

(2) One can check that the reduced expression $\mathbf{i}_{0}$ of type $A_{n}$ is adapted to Dynkin quiver of type $\mathrm{A}_{n}$ with a single sink (cf. [27]), while the one of the other types does not satisfy the adaptedness.

3.3. Positive roots and convex order. From now on, we assume that $\mathfrak{g}$ is of type $\mathrm{E}_{6}$ or $\mathrm{E}_{7}$, where the Dynkin diagram is given in Figure 1, In this subsection, we consider the positive roots and the convex order determined by $\mathbf{i}_{0}$ in the sense of (3.1).

3.3.1. Type $\mathbf{E}_{\mathbf{6}}$. In this case, we only consider the case of $r=6$ since the case of $r=1$ is almost identical. For simplicity, we denote by $\underset{b c d e f}{b}$ the positive root $a \alpha_{1}+b \alpha_{2}+c \alpha_{3}+d \alpha_{4}+e \alpha_{5}+f \alpha_{6} \in \Phi^{+}$. Then the convex order associated to $\mathbf{i}_{0}$ is given as follows.

$$
\begin{aligned}
& \underset{00001}{0}<\underset{00011}{0}<\underset{00111}{0}<\underset{01111}{0}<\underset{11111}{0}<00111<{ }_{01111}^{1}<{ }_{11111}^{1} \\
& <\underset{01211}{1}<\underset{11211}{1}<\underset{12211}{1}<\underset{01221}{1}<\frac{11221}{1}<\underset{12221}{1}<\underset{12321}{1}<\frac{12321}{2}
\end{aligned}
$$

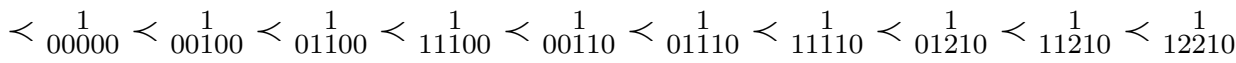

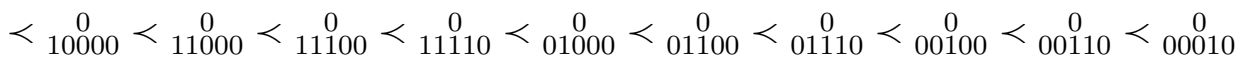

where $\underset{12321}{2}$ is the maximal root of $\mathfrak{g}$. Then let us introduce an arrangement of dots in the plane to present the positive roots following the above convex order. More precisely, we associate the $k$-th dot in the arrangement in (3.7) with the $k$-th positive 
root in (3.6) along the convex order. Then we often identify the arrangement in (3.7) with the one of the above positive roots.

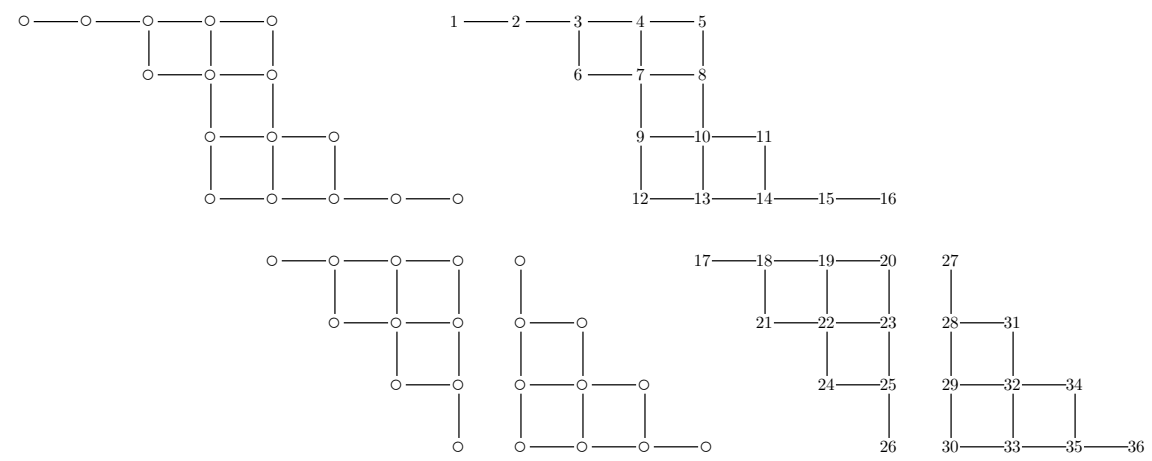

In particular, we will focus on the part consisting of the positive roots defined as in (2.2) with respect to $\mathrm{i}^{\mathrm{J}}$, which is denoted by $\Delta_{6,6}^{\mathrm{J}}$ (see (3.8) ). For the case of $r=1$, we also denote the arrangement by $\Delta_{6,1}^{\mathrm{J}}$ similarly. Note that $\Delta_{6,1}^{\mathrm{J}}$ may be obtained from $\Delta_{6,6}^{\mathrm{J}}$ by replacing $6 \leftrightarrow 1$ and $5 \leftrightarrow 3$.

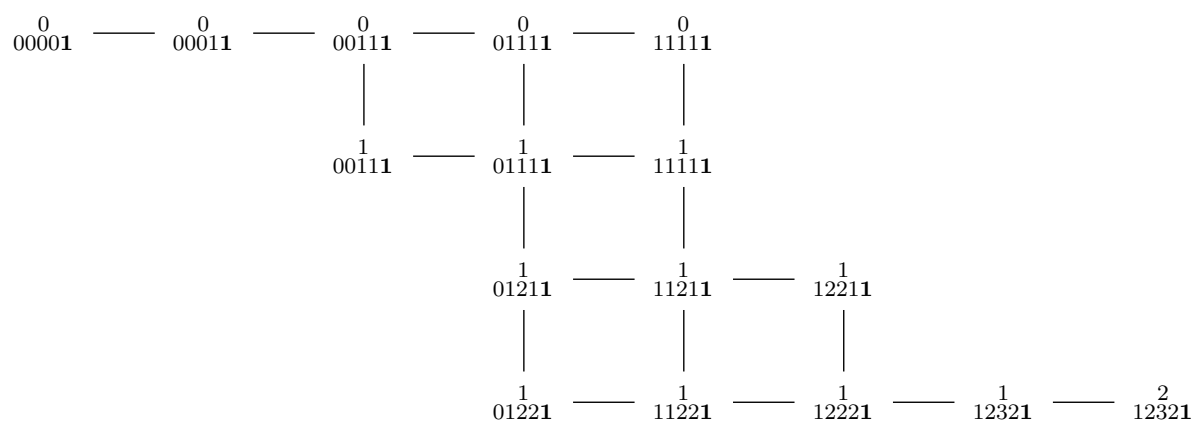

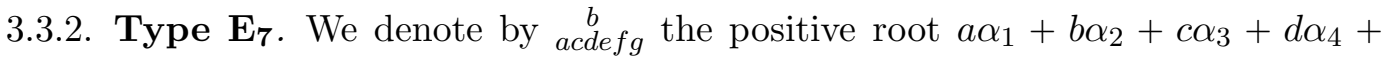
$e \alpha_{5}+f \alpha_{6}+g \alpha_{7} \in \Phi^{+}$. Then the convex order associated to $\mathbf{i}_{0}$ is given as follows.

$$
\begin{aligned}
& { }_{000001}^{0}<{ }_{000011}^{0}<\underset{000111}{0}<{ }_{001111}^{0}<{ }_{011111}^{0}<{ }_{111111}^{0}<{ }_{001111}^{1}<{ }_{011111}<{ }_{111111}^{1}
\end{aligned}
$$

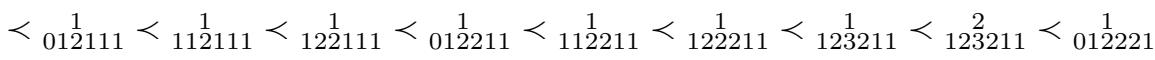

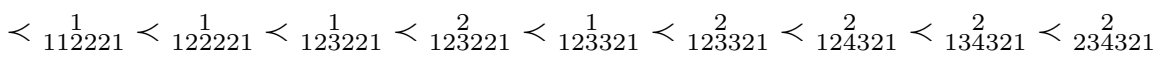

$$
\begin{aligned}
& <\ldots
\end{aligned}
$$

where $\underset{234321}{2}$ is the maximal root of $\mathfrak{g}$. Here the above positive roots are associated to $\mathbf{i}^{\mathrm{J}}$ and the remaining part coincides with (3.6) under the identification $\stackrel{b}{b}$ acdef0 with $\underset{a c d e f}{b}$. As in type $\mathrm{E}_{6}$, let us introduce the arrangement of dots in the plane to present the positive roots, and identify the arrangement with the one of the positive roots 
along the above convex order. Also, we denote by $\Delta_{7}^{\mathrm{J}}$ the part of that corresponding to the positive roots defined as in (2.2) with respect to $\mathbf{i}^{\mathrm{J}}$ (see (3.10) $)$.

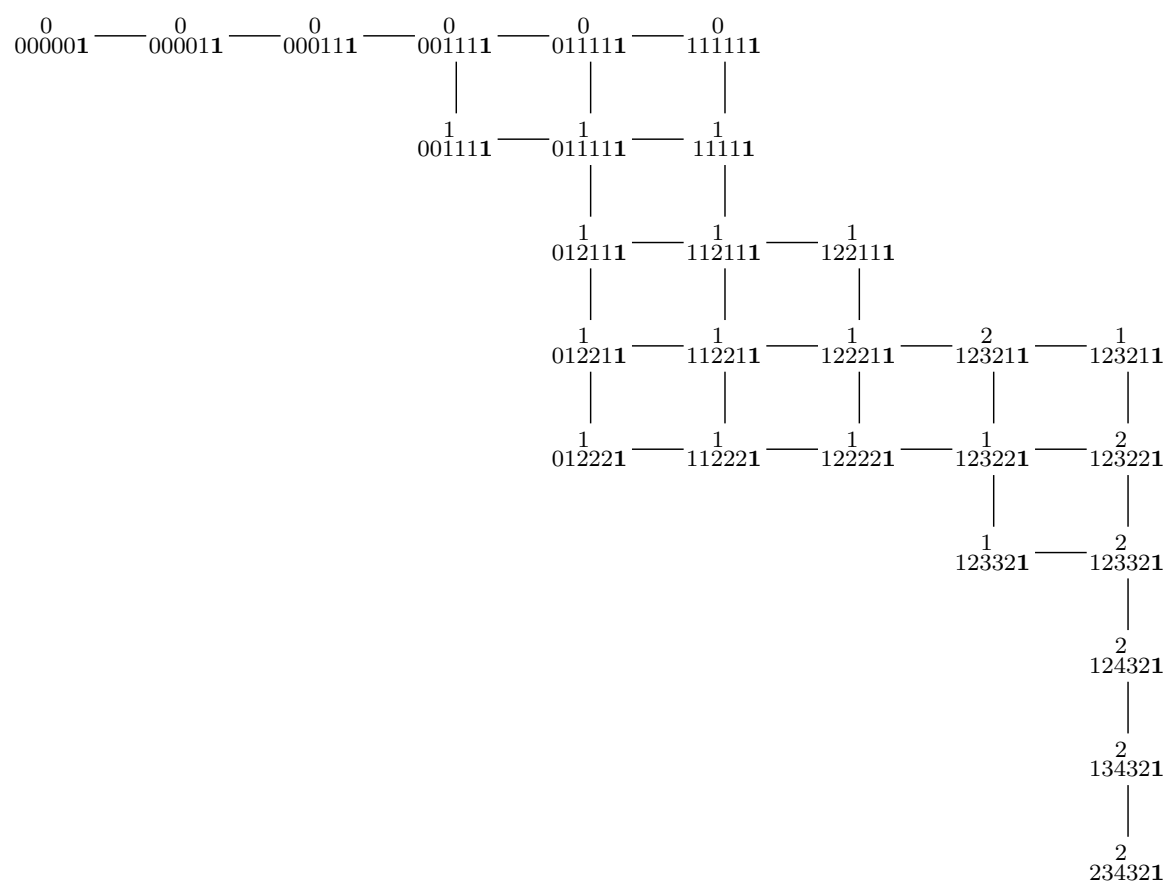

3.3.3. The arrangement $\Delta_{n}$. Let us consider the arrangement $\Delta_{n}$ of dots in the plane to present $(n-1)$-th triangular number. We often denote by $(i, j)$ the position of a dot under the numbering on rows and columns. For example, $\Delta_{10}$ is given by

\begin{tabular}{|c|c|c|c|c|c|c|c|c|}
\hline & 9 & 8 & 7 & 6 & 5 & 4 & 3 & 2 \\
\hline 1 & 0 & 0 & 0 & 0 & 0 & 0 & $\circ$ & 0 \\
\hline 2 & & 0 & 0 & 0 & 0 & 0 & 0 & 0 \\
\hline 3 & & & 0 & 0 & 0 & 0 & 0 & 0 \\
\hline 4 & & & & 0 & 0 & 0 & 0 & 0 \\
\hline 5 & & & & & 0 & 0 & o & o \\
\hline 6 & & & & & & 0 & 0 & ○ \\
\hline 7 & & & & & & & 0 & 0 \\
\hline 8 & & & & & & & & 0 \\
\hline
\end{tabular}


Then we often regard $\Delta_{6, r}^{\mathrm{J}}(r=1,6)$ and $\Delta_{7}^{\mathrm{J}}$ as the sub-arrangements of $\Delta_{9}$ and $\Delta_{10}$, respectively, that is,

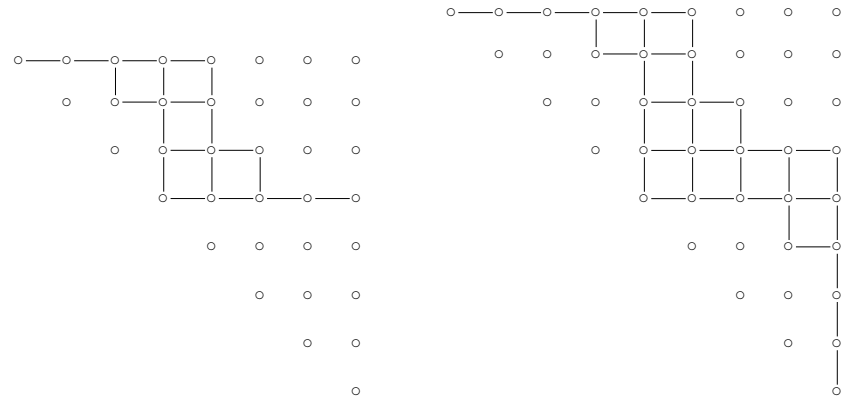

This convention will be used in Section [6 to define a certain statistic on $\Delta_{6, r}^{\mathrm{J}}$ and $\Delta_{7}^{\mathrm{J}}$ to present a combinatorial formula of $\varepsilon_{r}^{*}$.

Remark 3.5. If there is no confusion on $\mathfrak{g}$ and $r$, then we write the arrangements $\Delta_{6, r}^{\mathrm{J}}$ and $\Delta_{7}^{\mathrm{J}}$ by $\Delta^{\mathrm{J}}$ simply.

\section{Crystals of the Quantum nilpotent subalgebras of type E}

4.1. Description of $\tilde{f}_{i}$. Let us briefly review the result in [40. For simplicity, we assume that $\mathfrak{g}$ is simply laced. Let $\mathbf{i} \in R\left(w_{0}\right)$ be given. For $\beta \in \Phi^{+}$, we denote by $\mathbf{1}_{\beta}$ the element in $\mathbf{B}_{\mathbf{i}}$ where $c_{\beta}=1$ and $c_{\gamma}=0$ for $\gamma \in \Phi^{+} \backslash\{\beta\}$.

Let $\sigma=\left(\sigma_{1}, \sigma_{2}, \ldots, \sigma_{s}\right)$ be a sequence with $\sigma_{u} \in\{+,-, \cdot\}$. We replace a pair $\left(\sigma_{u}, \sigma_{u^{\prime}}\right)=(+,-)$, where $u<u^{\prime}$ and $\sigma_{u^{\prime \prime}}=$. for $u<u^{\prime \prime}<u^{\prime}$, with $(\cdot, \cdot)$, and repeat this process as far as possible until we get a sequence with no - placed to the right of + . We denote the resulting sequence by $\sigma^{\text {red }}$. For another sequence $\tau=\left(\tau_{1}, \ldots, \tau_{t}\right)$, we denote by $\sigma \cdot \tau$ the concatenation of $\sigma$ and $\tau$.

Given $i \in \mathrm{I}$, suppose that $\mathbf{i}$ is simply braided for $i \in \mathrm{I}$ (recall Definition 3.1). Then one can obtain $\mathbf{i}^{\prime}=\left(i_{1}^{\prime}, \ldots, i_{N}^{\prime}\right) \in R\left(w_{0}\right)$ with $i_{1}^{\prime}=i$ by applying a sequence of braid moves consisting of either a 2 -term move or 3 -term braid move $\left(\gamma, \gamma^{\prime}, \gamma^{\prime \prime}\right) \rightarrow\left(\gamma^{\prime \prime}, \gamma^{\prime}, \gamma\right)$ with $\gamma^{\prime \prime}=\alpha_{i}$. Let

$$
\Pi_{s}=\left\{\gamma_{s}, \gamma_{s}^{\prime}, \gamma_{s}^{\prime \prime}\right\}
$$

be the triple of positive roots of type $A_{2}$ with $\gamma_{s}^{\prime}=\gamma_{s}+\gamma_{s}^{\prime \prime}$ and $\gamma_{s}^{\prime \prime}=\alpha_{i}$ corresponding to the $s$-th 3 -term braid move for $1 \leqslant s \leqslant t$. For $\mathbf{c} \in \mathbf{B}_{\mathbf{i}}$, let

$$
\sigma_{i}(\mathbf{c})=(\underbrace{-\cdots}_{c_{\gamma_{1}^{\prime}}} \underbrace{+\cdots+}_{c_{\gamma_{1}}} \cdots \underbrace{-\cdots}_{c_{\gamma_{t}^{\prime}}} \underbrace{+\cdots+}_{c_{\gamma_{t}}}) .
$$

Theorem 4.1. [40, Theorem 4.6] Let $\mathbf{i} \in R\left(w_{0}\right)$ and $i \in \mathrm{I}$. Suppose that $\mathbf{i}$ is simply braided for $i$. Let $\mathbf{c} \in \mathbf{B}_{\mathbf{i}}$ be given. 
(1) If there exists + in $\sigma_{i}(\mathbf{c})^{\text {red }}$ and the leftmost + appears in $c_{\gamma_{s}}$, then

$$
\tilde{f}_{i} \mathbf{c}=\mathbf{c}-\mathbf{1}_{\gamma_{s}}+\mathbf{1}_{\gamma_{s}^{\prime}} .
$$

(2) If there exists no + in $\sigma_{i}(\mathbf{c})^{\text {red }}$, then

$$
\tilde{f}_{i} \mathbf{c}=\mathbf{c}+\mathbf{1}_{\alpha_{i}} .
$$

4.2. Crystal $\mathbf{B}^{\mathbf{J}}$ of the quantum nilpotent subalgebra. In this subsection, we consider the crystal $\mathbf{B}^{\mathrm{J}}$ of the quantum nilpotent subalgebra $U_{q}\left(w^{\mathrm{J}}\right)$, which is obtained as a subcrystal of $\mathbf{B}_{\mathbf{i}_{0}}$, where $\mathbf{i}_{0}$ is the reduced expression of $w_{0}$ given in (3.2). Throughout this paper, we set

$$
\mathbf{B}=\mathbf{B}_{\mathbf{i}_{0}} .
$$

Recall $\mathrm{J}=\mathrm{I} \backslash\{r\}$. Let $\mathfrak{l}$ be the maximal Levi subalgebra of $\mathfrak{g}$ associated to $\left\{\alpha_{i} \mid i \in\right.$ $J\}$ of types $\mathrm{D}_{5}$ and $\mathrm{E}_{6}$, respectively, as shown on the following Dynkin diagrams:

$\mathrm{D}_{5}$

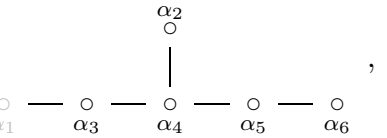

$\mathrm{E}_{6}$

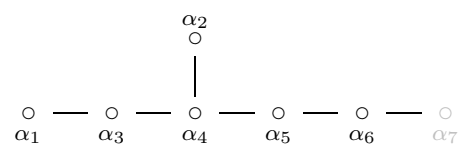

Figure 2. For type $\mathrm{E}_{6}$ (resp. $\mathrm{E}_{7}$ ), the maximal Levi subalgebra $\mathfrak{l}$ is of type $\mathrm{D}_{5}$ (resp. $\left.\mathrm{E}_{6}\right)$.

Let $\Phi_{\mathrm{J}}^{+}$be the set of positive roots of $\mathfrak{l}$ and let $\Phi^{+}(\mathrm{J})$ be the set of positive roots of the nilradical $\mathfrak{u}$ of the parabolic subalgebra of $\mathfrak{g}$ associated to $\mathfrak{l}$. Note that

$$
\Phi^{+}=\Phi^{+}(\mathrm{J}) \sqcup \Phi_{\mathrm{J}}^{+},
$$

and the positive roots in $\Phi^{+}(\mathrm{J})$ coincide with the ones in (3.8) and (3.10).

Remark 4.2. We should remark that $\tilde{f}_{i}$ on $\mathbf{B}$ can be described by using Theorem 4.1 due to Proposition 3.3 in the sense of [16, Remark 3.3] (cf. [40, Remark 4.6]).

For simplicity, we modify the notation (4.1) for type E. For example, if $\mathfrak{g}$ is of type $\mathrm{E}_{6}$ with $\gamma_{s}={ }_{11100}^{0}$ and $\gamma_{s}^{\prime}={ }_{11100}^{1}$, then we write

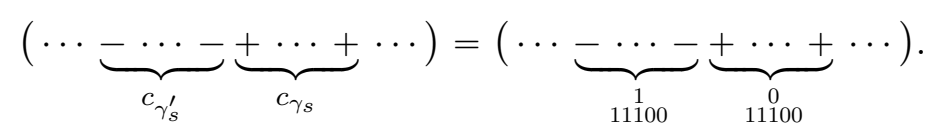

Proposition 4.3. Let $i \in \mathrm{I}$ and $\mathbf{c} \in \mathbf{B}_{\mathbf{i}}$ be given, where $i \neq r$ and $\mathbf{i}$ is in Theorem 3.3. Then the sequence (4.1) is given as follows. 
(1) in type $\mathrm{E}_{6}$ with $r=1$, we have

$$
\sigma_{i}(\mathbf{c})=\sigma_{i, 1}(\mathbf{c}) \cdot \sigma_{i, 2}(\mathbf{c})
$$

where $\sigma_{i, 1}(\mathbf{c})$ is given by

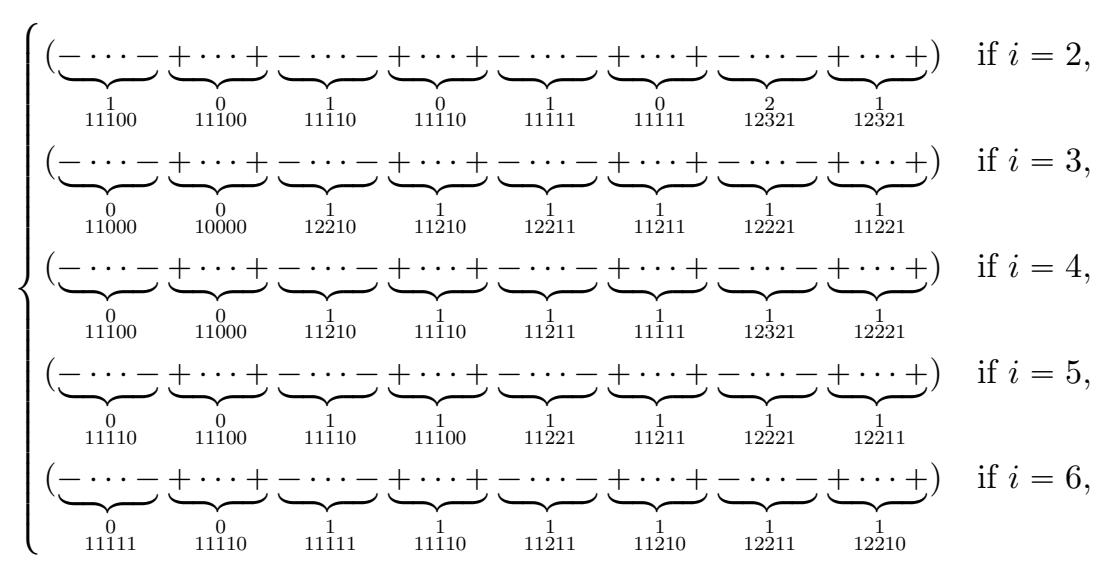

and $\sigma_{i, 2}(\mathbf{c})$ is equal to the sequence for $k$ such that $k^{\star}=i$ obtained from [16, Proposition 3.2] to the pair $\left(\mathfrak{l}, \mathbf{i}_{\mathrm{J}}\right)$ by regarding $\underset{0 c d e f}{b}$ as the root of type $\mathrm{D}_{5}$.

(2) in type $\mathrm{E}_{6}$ with $r=6$, we have

$$
\sigma_{i}(\mathbf{c})=\sigma_{i, 1}(\mathbf{c}) \cdot \sigma_{i, 2}(\mathbf{c})
$$

where $\sigma_{i, 1}(\mathbf{c})$ is given by

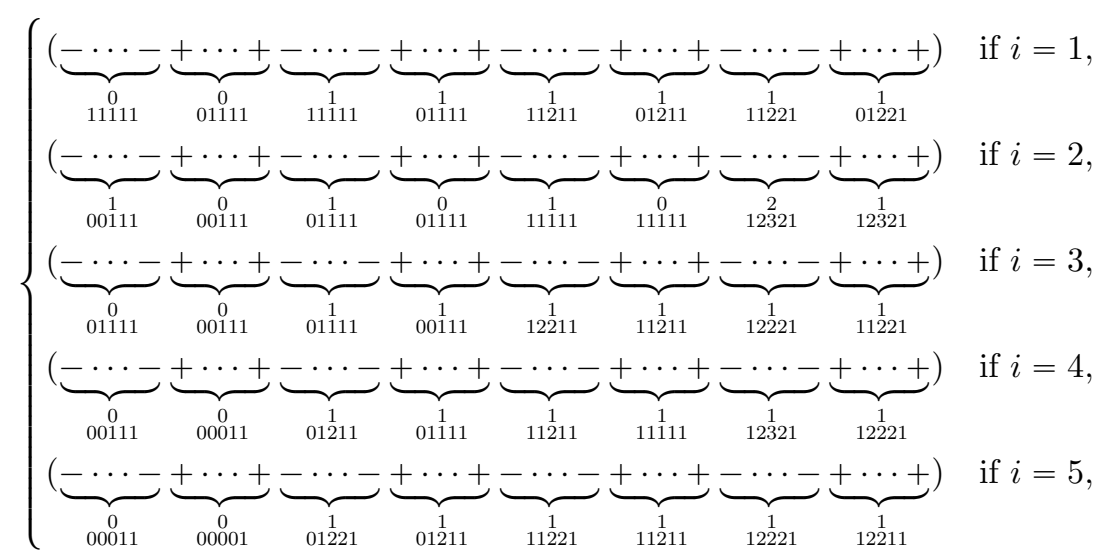

and $\sigma_{i, 2}(\mathbf{c})$ is equal to the sequence for $k$ such that $k^{\star}=i$ obtained from [16, Proposition 3.2] to the pair $\left(\mathfrak{l}, \mathbf{i}_{\mathrm{J}}\right)$ by regarding $\underset{\text { acde0 }}{b}$ as the root of type $\mathrm{D}_{5}$.

(3) in type $\mathrm{E}_{7}$ with $r=7$, we have

$$
\sigma_{i}(\mathbf{c})=\sigma_{i, 1}(\mathbf{c}) \cdot \sigma_{i, 2}(\mathbf{c})
$$


where $\sigma_{i, 1}(\mathbf{c})$ is given by

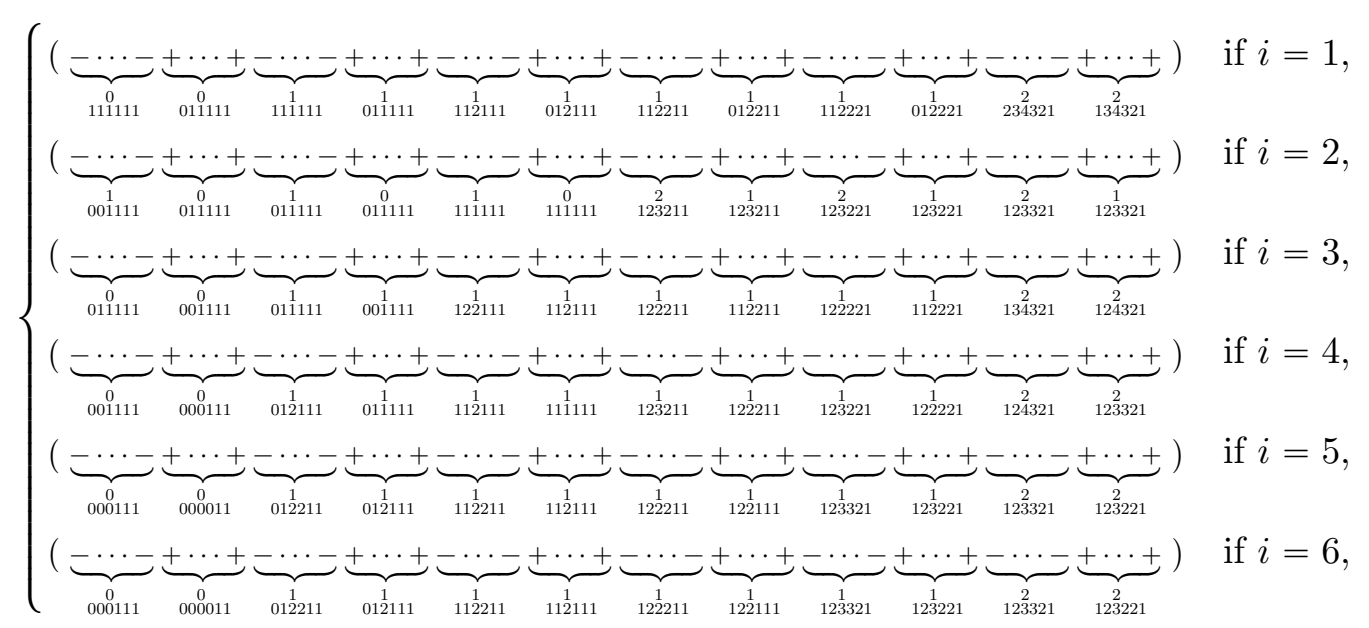

and $\sigma_{i, 2}(\mathbf{c})$ is equal to the sequence $\sigma_{k}(\mathbf{c})$ for $k$ such that $k^{\star}=i$ in (2) by regarding $\underset{\text { acdef } 0}{b}$ as the root of type $\mathrm{E}_{6}$.

Proof. By Theorem 4.1 and Remark 4.2 , the sequence $\sigma_{i}(\mathbf{c})$ is obtained from $\overline{\mathbf{i}}_{k}^{\mathrm{J}} \cdot \mathbf{i}_{\mathrm{J}}$ such that $k^{\star}=i$ (cf. (3.4) and (3.5)).

Set

$$
\begin{aligned}
& \mathbf{B}^{\mathrm{J}}=\left\{\mathbf{c}=\left(c_{\beta}\right) \in \mathbf{B} \mid c_{\beta}=0 \text { unless } \beta \in \Phi^{+}(\mathrm{J})\right\}, \\
& \mathbf{B}_{\mathrm{J}}=\left\{\mathbf{c}=\left(c_{\beta}\right) \in \mathbf{B} \mid c_{\beta}=0 \text { unless } \beta \in \Phi_{\mathrm{J}}^{+}\right\},
\end{aligned}
$$

which we regard them as subcrystals of $\mathbf{B}$, where we assume that $\tilde{e}_{r} \mathbf{c}=\tilde{f}_{r} \mathbf{c}=\mathbf{0}$ with $\varepsilon_{r}(\mathbf{c})=\varphi_{r}(\mathbf{c})=-\infty$ for $\mathbf{c} \in \mathbf{B}_{\mathrm{J}}$. Then the crystal $\mathbf{B}^{\mathrm{J}}$ can be viewed as the crystal of the quantum nilpotent subalgebra $U_{q}\left(w^{\mathrm{J}}\right)$.

Remark 4.4. The crystal operators $\tilde{e}_{i}$ and $\tilde{f}_{i}(i \in \mathrm{J})$ on $\mathbf{B}^{\mathrm{J}}$ and $\mathbf{B}_{\mathrm{J}}$ are described by the rule in Theorem 4.1 with respect to the sequences $\sigma_{i, 1}$ and $\sigma_{i, 2}$ in Proposition 4.3, respectively. Note that it is well-known in [34] (cf. [20, 39]) that for $\mathbf{c} \in \mathbf{B}$,

$$
\tilde{e}_{r} \mathbf{c}=\left\{\begin{array}{ll}
\mathbf{c}-\mathbf{1}_{\alpha_{r}} & \text { if } c_{\alpha_{r}} \neq 0, \\
\mathbf{0} & \text { otherwise },
\end{array} \quad \tilde{f}_{r} \mathbf{c}=\mathbf{c}+\mathbf{1}_{\alpha_{r}},\right.
$$

since $\beta_{1}$ in the convex order of $\mathbf{i}_{0}$ is equal to $\alpha_{r}$.

Remark 4.5. For $\mathbf{c}=\left(c_{\beta_{k}}\right) \in \mathbf{B}^{\mathrm{J}}$, we often identify it with an array of $c_{\beta_{k}}$ 's located at the position of $\beta_{k}$ on $\Delta^{\mathrm{J}}$ (recall (3.8) and (3.10) $)$. Then the sequence $\sigma_{i}(\mathbf{c})$ for $\tilde{f}_{i}$ $(i \neq r)$ is recorded easily under this identification. Let us explain it in more details. 
For type $\mathrm{E}_{6}$ with $r=6$, the array is given by

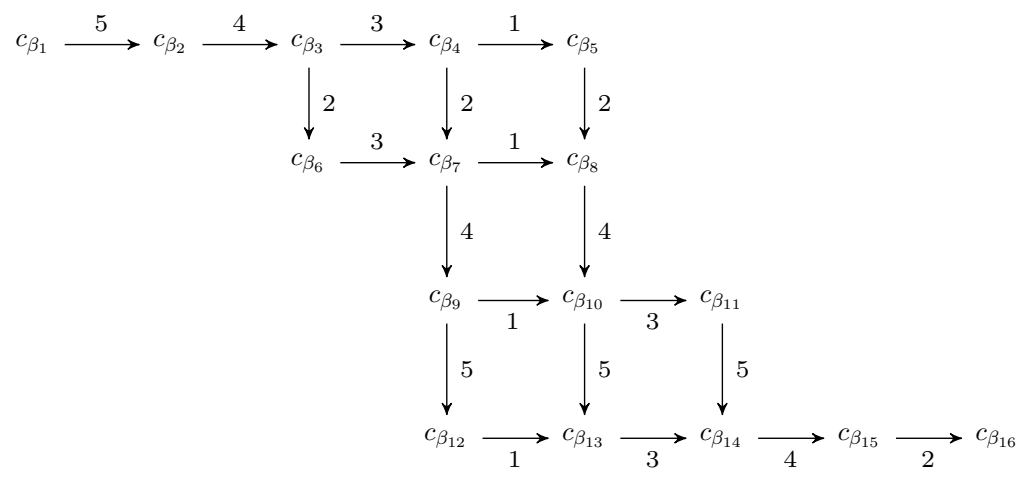

where $\beta_{k}$ 's are enumerated by the convex ordering (3.6) and the arrow is determined by $c_{\beta} \stackrel{i}{\longrightarrow} c_{\beta^{\prime}}$ if and only if $\beta^{\prime}-\beta=\alpha_{i}$. The sequence $\sigma_{i}(\mathbf{c})$ is obtained by reading -'s and + 's as $c_{\beta_{k}}$ located in the terminal and initial points of the arrows labelled by $i$ from north-west to south-east. The sequences $\sigma_{i}(\mathbf{c})$ for the case of $r=1$ are also given by the similar rule.

For type $\mathrm{E}_{7}$, the array for $\mathbf{c} \in \mathbf{B}^{\mathrm{J}}$ with the arrows labelled by $i(i \in \mathrm{J})$ is given by

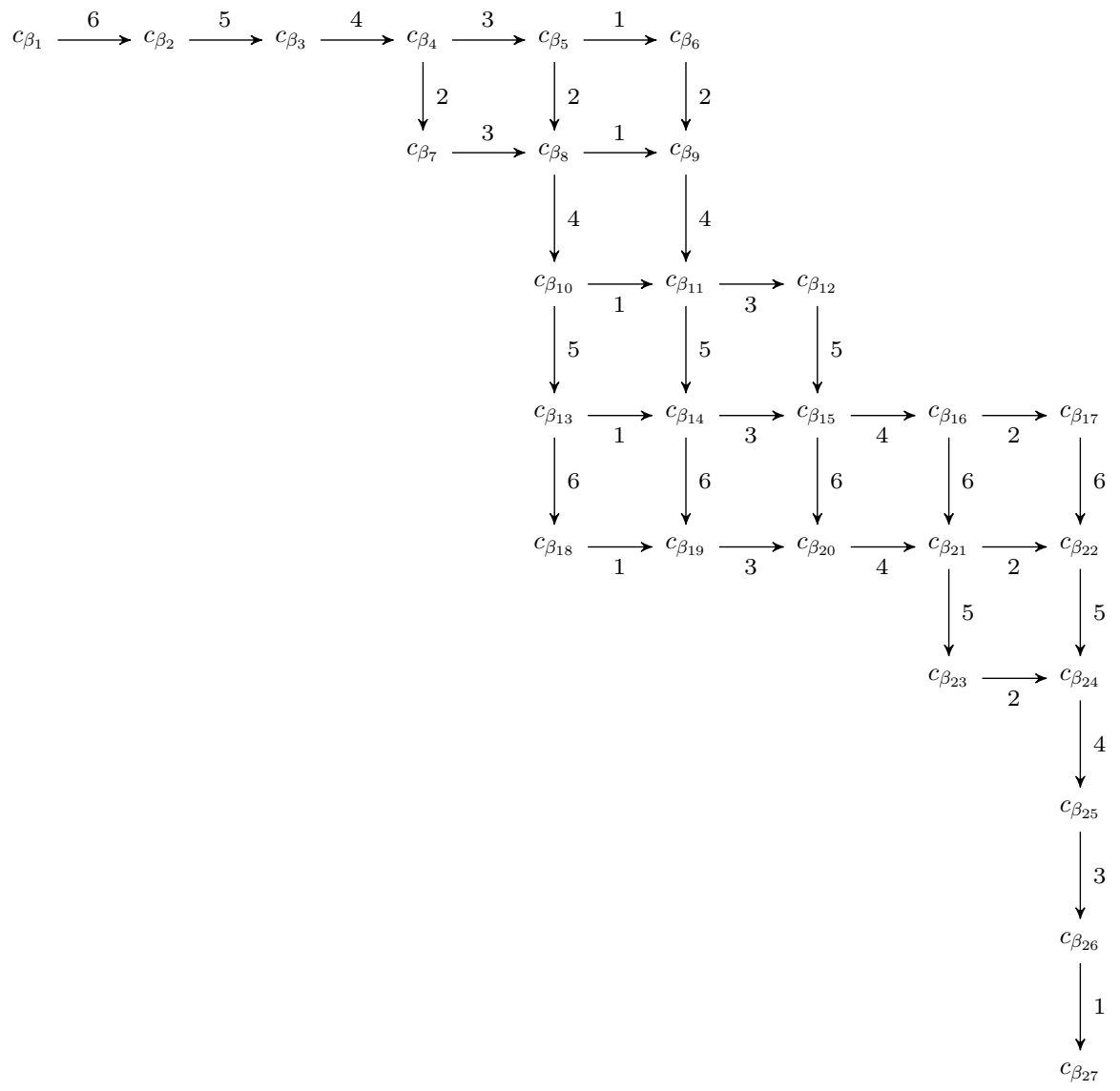


where $\beta_{k}$ 's are enumerated by the convex ordering (3.9).

The following is the type E analog of [27, Theorem 4.2] (cf. [16, Corollary 3.5] for type D).

\section{Corollary 4.6.}

(1) The crystal $\mathbf{B}_{\mathrm{J}}$ is isomorphic to the crystal of $U_{q}^{-}(\mathfrak{l})$ as an $\mathfrak{l}$-crystal.

(2) The crystal $\mathbf{B}$ is isomorphic to $\mathbf{B}^{\mathrm{J}} \otimes \mathbf{B}_{\mathrm{J}}$ as an $\mathfrak{g}$-crystal by the map $\mathbf{c} \mapsto \mathbf{c}^{\mathrm{J}} \otimes \mathbf{c}_{\mathrm{J}}$.

Proof. (1) It follows from comparing the crystal structure of $U_{q}^{-}(\mathfrak{l})$ given in [16, Section 3.1].

(2) It follows from Theorem 4.1, Proposition 4.3 and the tensor product rule (2.1).

We have the characterizations of $\mathbf{B}^{\mathrm{J}}$ and $B\left(s \varpi_{r}\right)$ for $s \geqslant 1$ by using the $\varepsilon_{i}^{*}$ statistics as follows.

\section{Proposition 4.7.}

(1) We have

$$
\mathbf{B}^{\mathrm{J}}=\left\{\mathbf{c} \in \mathbf{B} \mid \varepsilon_{i}^{*}(\mathbf{c})=0 \text { for } i \in \mathrm{J}\right\} .
$$

(2) For $s \geqslant 1$, let

$$
\mathbf{B}^{\mathrm{J}, s}:=\left\{\mathbf{c} \in \mathbf{B}^{\mathrm{J}} \mid \varepsilon_{r}^{*}(\mathbf{c}) \leqslant s\right\}
$$

and it is regarded as a subcrystal of $\mathbf{B}^{\mathrm{J}}$. As $\mathfrak{g}$-crystals, we have

$$
\mathbf{B}^{\mathrm{J}, s} \otimes T_{s \varpi_{r}} \cong B\left(s \varpi_{r}\right), \quad \mathbf{B}^{\mathrm{J}}=\bigcup_{s \geqslant 1} \mathbf{B}^{\mathrm{J}, s} .
$$

Proof. (1) follows from [32, Section 2.1], and (2) follows from (1) and 21, Proposition $8.2]$.

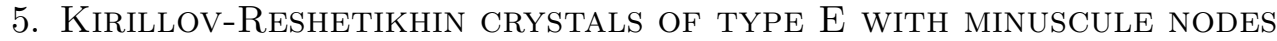

Let $\hat{\mathfrak{g}}$ be the affine Lie algebra of types $\mathrm{E}_{6}^{(1)}$ or $\mathrm{E}_{7}^{(1)}$ with the index set $\hat{\mathrm{I}}$ and the Dynkin diagram given by

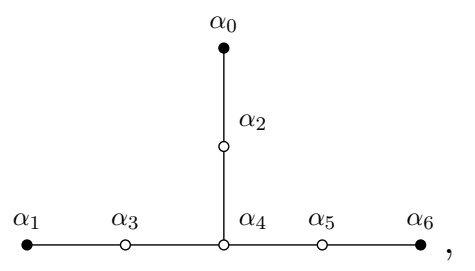

Type $\mathrm{E}_{6}^{(1)}$

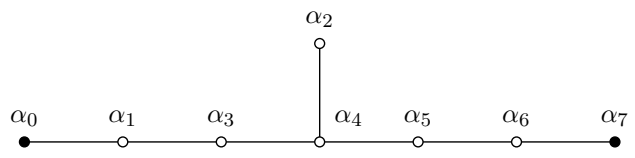

Type $\mathrm{E}_{7}^{(1)}$ 
For $k$ in the orbit of 0 , that is,

$$
k \in \begin{cases}\{0,1,6\} & \text { for type } \mathrm{E}_{6}^{(1)}, \\ \{0,7\} & \text { for type } \mathrm{E}_{7}^{(1)},\end{cases}
$$

let $\hat{\mathfrak{g}}_{k}$ be the subalgebra of $\hat{\mathfrak{g}}$ corresponding to $\left\{\alpha_{i} \mid i \in \hat{\mathrm{I}} \backslash\{k\}\right\}$. Note that $\hat{\mathfrak{g}}_{0}=\mathfrak{g}$ and $\hat{\mathfrak{g}}_{0} \cap \hat{\mathfrak{g}}_{k}=\mathfrak{l}$ for $k \neq 0$. Let $\hat{P}=\bigoplus_{i \in \hat{\mathrm{I}}} \mathbb{Z} \Lambda_{i} \oplus \mathbb{Z} \delta$ be the weight lattice of $\hat{\mathfrak{g}}$, where $\delta$ is the positive imaginary null root and $\Lambda_{i}$ is the $i$ th fundamental weight of $\hat{\mathfrak{g}}$ [19]. Let $\theta$ be the maximal root of $\mathfrak{g}$. Then $\alpha_{0}=-\theta$ in $\hat{P} / \mathbb{Z} \delta$.

For $\mathbf{c} \in \mathbf{B}^{\mathrm{J}}$, we define

$$
\begin{aligned}
& \tilde{e}_{0} \mathbf{c}=\mathbf{c}+\mathbf{1}_{\theta}, \quad \tilde{f}_{0} \mathbf{c}= \begin{cases}\mathbf{c}-\mathbf{1}_{\theta} & \text { if } c_{\theta}>0, \\
\mathbf{0} & \text { otherwise, }\end{cases} \\
& \varphi_{0}(\mathbf{c})=\max \left\{k \mid \tilde{f}_{0}^{k} \mathbf{c} \neq 0\right\}, \quad \varepsilon_{0}(\mathbf{c})=\varphi_{0}(\mathbf{c})-\left\langle\operatorname{wt}(\mathbf{c}), h_{0}\right\rangle .
\end{aligned}
$$

The set $\mathbf{B}^{\mathrm{J}}$ is a $\hat{\mathfrak{g}}$-crystal with respect to wt, $\varepsilon_{i}, \varphi_{i}, \tilde{e}_{i}, \tilde{f}_{i}$ for $i \in \hat{\mathrm{I}}$, where wt is the restriction of wt : $\mathbf{B} \rightarrow P$ to $\mathbf{B}^{\mathrm{J}}$, and then we regard the subcrystal $\mathbf{B}^{\mathrm{J}, s}$ of $\mathbf{B}^{\mathrm{J}}$ as a $\hat{\mathfrak{g}}$-crystal.

Now we are in position to state the main result in this paper.

Theorem 5.1. For a minuscule $\varpi_{r}$ and $s \geqslant 1$, the $\hat{\mathfrak{g}}$-crystal $\mathbf{B}^{\mathrm{J}}$ is regular and

$$
\mathbf{B}^{\mathrm{J}, s} \otimes T_{s \varpi_{r}} \cong B^{r, s},
$$

where $B^{r, s}$ is the $\mathrm{KR}$ crystal of types $\mathrm{E}_{6}^{(1)}$ and $\mathrm{E}_{7}^{(1)}$ associated to $s \varpi_{r}$.

Proof. By Proposition $4.7(2), \mathbf{B}^{\mathrm{J}, s} \otimes T_{s \varpi_{r}}$ is a regular $\hat{\mathfrak{g}}_{0}$-crystal isomorphic to $B\left(s \varpi_{r}\right)$. By Proposition 4.3 (see also Remark 4.5), one can check that the $\hat{\mathfrak{g}}_{r^{-}}$ crystal $\mathbf{B}^{\mathrm{J}, s} \otimes T_{s \varpi_{r}}$ is isomorphic to the dual crystal of $\hat{\mathfrak{g}}_{0}$-crystal $\mathbf{B}^{\mathrm{J}, s} \otimes T_{s \varpi_{r}}$ by regarding $\hat{\mathfrak{g}}_{r} \cong \hat{\mathfrak{g}}_{0}$ under the following correspondence

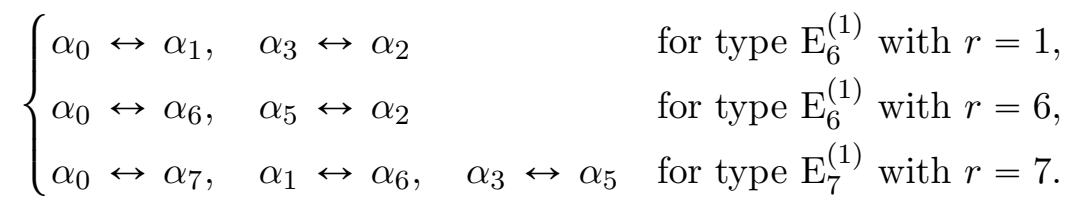

This implies that the $\hat{\mathfrak{g}}_{r}$-crystal $\mathbf{B}^{\mathrm{J}, s} \otimes T_{s \varpi_{r}}$ is isomorphic to $B\left(s \varpi_{r} *\right)$ under the above correspondence, where $r^{*}$ is determined by $w_{0}\left(\alpha_{r}\right)=-\alpha_{r} *$ (cf. [4]). It was well-known in [6] that $B^{r, s}$ is classically irreducible, that is, $B^{r, s} \cong B\left(s \varpi_{r}\right)$ as a

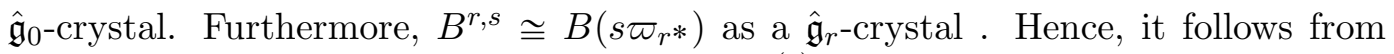
[18, Theorem 3.15] (see Remark [5.2 for type $\mathrm{E}_{7}^{(1)}$ ) that $\mathbf{B}^{\mathrm{J}, s} \otimes T_{s \varpi_{r}}$ is isomorphic to $B^{r, s}$ as a $\hat{\mathfrak{g}}$-crystal. 
Remark 5.2. Recall that $\mathfrak{l}$ is of type $E_{6}$ when $\hat{\mathfrak{g}}$ is of type $E_{7}^{(1)}$. Since the $\mathfrak{l}$-highest weight vectors $b$ in $B^{7, s}$ are distinguished by their weight with the value $\left\langle\operatorname{wt}(b), \alpha_{7}^{v}\right\rangle$ [1, Section 3.4], it allows us to obtain an analog of [18, Theorem 3.15] for type $\mathrm{E}_{7}^{(1)}$ with $r=7$ by following the proof of [18, Theorem 3.15] with $K=\hat{\mathrm{I}} \backslash\{0,7\}$ since $\left\langle\operatorname{wt}(b), \alpha_{7}^{\vee}\right\rangle=\left\langle\operatorname{wt}(b), \alpha_{0}^{\vee}\right\rangle$ for a l-highest weight vector $b$ by the level-zero condition (cf. [18, Remark 3.4]).

Remark 5.3. By Proposition 4.7 and Theorem [5.1, the crystal $\mathbf{B}^{\mathrm{J}}$ can viewed as the limit of KR crystals $B^{r, s}$ as $s \rightarrow \infty$.

Remark 5.4. After this paper was submitted, Hiroshima in [13] proved the perfectness of $\mathrm{KR}$ crystal $B^{r, s}$ for a minuscule $r$ and $s \geqslant 1$ in type $\mathrm{E}_{6,7}^{(1)}$ based on Theorem 5.1 .

\section{Combinatorial Description of the $\varepsilon_{r}^{*}$-STAtistic}

6.1. Combinatorial formula for $\varepsilon_{r}^{*}$. In this section, we give a combinatorial formula of the statistic $\varepsilon_{r}^{*}$ on $\mathbf{B}^{\mathrm{J}}$ for type $\mathrm{E}_{n}$ in terms of the triple or quadruple paths on $\Delta_{n+3}$, respectively.

Definition 6.1. (cf. [16, Definition 3.10]) Let $\beta$ be a dot in $\Delta_{n}$.

(1) A (single) path on $\Delta_{n}$ is a sequence $p=\left(\gamma_{1}, \ldots, \gamma_{s}\right)$ of dots in $\Delta_{n}$ for some $s \geqslant 1$ such that the position $\left(i_{k}, j_{k}\right)$ of $\gamma_{k}$ is equal to $\left(i_{k-1}+1, j_{k-1}\right)$ or $\left(i_{k-1}, j_{k-1}+1\right)$ for all $2 \leqslant k \leqslant s$.

(2) A double path at $\beta$ is a pair of paths $\mathbf{p}=\left(p_{1}, p_{2}\right)$ in $\Delta$ of the same length with $p_{1}=\left(\gamma_{1}, \ldots, \gamma_{s}\right)$ and $p_{2}=\left(\delta_{1}, \ldots, \delta_{s}\right)$ such that $\gamma_{1}=\delta_{1}=\beta$ and $\gamma_{i}$ is located strictly diagonally left of $\delta_{i}$ for $2 \leqslant i \leqslant s$.

(3) A triple path at $\beta$ is a sequence of paths $\mathbf{p}=\left(p_{1}, p_{2}, p_{3}\right)$ such that each pair $\left(p_{k}, p_{k+1}\right)$ is a double path at $\beta_{k}$ for $k=1,2$, where $\beta_{1}=\beta$ and $\beta_{2}$ is located strictly to the left of or below $\beta_{1}$ by one position.

(4) A quadruple path at $\beta$ is a sequence of paths $\mathbf{p}=\left(p_{1}, p_{2}, p_{3}, p_{4}\right)$ such that each pair $\left(p_{k}, p_{k+1}\right)$ is a double path at $\beta_{k}$ for $1 \leqslant k \leqslant 3$, where $\beta_{1}=\beta$ and $\beta_{k}$ is located strictly to the left of or below $\beta_{k-1}$ by one position for all $k=2,3$.

Definition 6.2. Let $\beta$ be the dot in $\Delta_{n+3}$ located at the position $(1,1)$. We denote by $p_{k}$ a path in $\Delta_{n+3}$ with $p_{k}=\left(\alpha_{1}^{(k)}, \ldots, \alpha_{s}^{(k)}\right)$ for $1 \leqslant k \leqslant 5$. We define $\mathcal{P}_{n}$ as follows.

(1) if $n=6$, then $\mathcal{P}_{6}$ is the set of the triple paths $\mathbf{p}=\left(p_{1}, p_{2}, p_{3}\right)$ at $\beta$ in $\Delta_{9}$ satisfying the following two conditions: 
(i) $\alpha_{s}^{(k+1)}$ is located strictly diagonally left of $\alpha_{s}^{(k)}$ by one position for all $k=1,2$,

(ii) $\alpha_{s}^{(1)}, \alpha_{s}^{(2)}$ and $\alpha_{s}^{(3)}$ are placed above the 4 -th row of $\Delta_{9}$ from top.

(2) if $n=7$, then $\mathcal{P}_{7}$ is the set of the quadruple paths $\mathbf{p}=\left(p_{1}, p_{2}, p_{3}, p_{4}\right)$ at $\beta$ in $\Delta_{10}$ passing through the dots located in $(4,1),(1,4)$ satisfying

(i) $\alpha_{s}^{(k+1)}$ is located strictly diagonally left of $\alpha_{s}^{(k)}$ by one position for all $1 \leqslant k \leqslant 3$,

(ii) $\alpha_{s}^{(1)}, \ldots, \alpha_{s}^{(4)}$ are placed above or below the 5 -th row of $\Delta_{10}$ from top, and the pairs $\left(\mathbf{p}_{1}, \mathbf{p}_{2}\right)$ of a double path $\mathbf{p}_{1}$ and a triple path $\mathbf{p}_{2}$ satisfying

(iii) $\mathbf{p}_{1}=\left(p_{1}, p_{2}\right)$ is a double path at $\beta^{\prime}$ such that $\alpha_{s}^{(1)}$ or $\alpha_{s}^{(2)}$ is placed at $(5,5)$, where $\beta^{\prime}$ is located at $(3,4)$ or $(4,3)$ in $\Delta_{10}$, and $\mathbf{p}_{2}=\left(p_{3}, p_{4}, p_{5}\right)$ is a triple path at $\beta$ such that $\mathbf{p}_{2}$ passes through the dots located at $(4,1),(1,4),(2,6)$ and $(6,2)$, while it does not pass through the dot located at $(3,3)$ in $\Delta_{10}$,

(iv) for $\left(\mathbf{p}_{1}, \mathbf{p}_{2}\right)$, the paths $\mathbf{p}_{1}$ and $\mathbf{p}_{2}$ are non-intersecting and $\left\{\alpha_{s}^{(k)} \mid 1 \leqslant\right.$ $k \leqslant 5\}$ satisfies the condition as in (1)-(i) under an enumeration such that $\alpha_{s}^{(1)}$ and $\alpha_{s}^{(2)}$ are placed between $\alpha_{s}^{(k)}$ for $3 \leqslant k \leqslant 5$ (cf. Example 6.3).

Example 6.3. The followings are examples of a triple path in $\mathcal{P}_{6}$, a quadruple path and a pair of double path and triple path in $\mathcal{P}_{7}$, respectively.
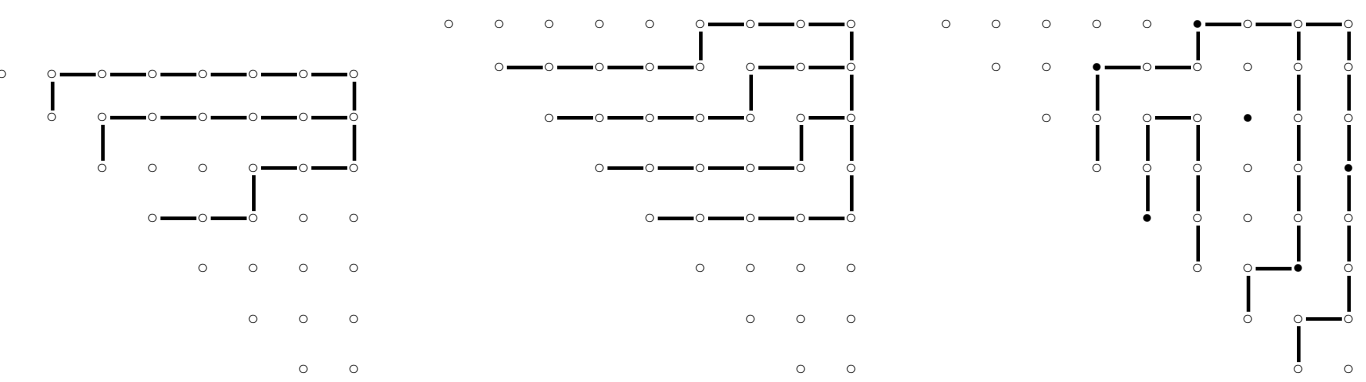

where the 's in the rightmost path are the dots located at $(4,1),(1,4),(2,6),(6,2),(5,5)$ and $(3,3)$, respectively. Note that by the condition (iv) in Definition 6.2(2), the triple path in a pair of double and triple paths in $\mathcal{P}_{7}$, does not pass through the dots located at $(1,9)$ and $(9,1)$. 
Put $\Delta=\Delta_{n+3}$. For $\mathbf{c} \in \mathbf{B}^{\mathrm{J}}$ and $\mathbf{p} \in \mathcal{P}_{n}(n=6,7)$, we define

$$
\|\mathbf{c}\|_{\mathbf{p}}=\sum_{\beta \text { lying on } \mathbf{p} \cap \Delta^{\mathrm{J}}} c_{\beta}
$$

where $\mathbf{p} \cap \Delta^{\mathrm{J}}$ is the set of the positive roots in $\Delta^{\mathrm{J}}$ lying on the path $\mathbf{p}$ under the identification of $\Delta^{\mathrm{J}}$ in $\Delta$.

Theorem 6.4. For $\mathbf{c} \in \mathbf{B}^{\mathrm{J}}$, we have

$$
\varepsilon_{r}^{*}(\mathbf{c})=\max \left\{\|\mathbf{c}\|_{\mathbf{p}} \mid \mathbf{p} \in \mathcal{P}_{n}\right\},
$$

where $\mathfrak{g}$ is of type $\mathrm{E}_{n}$ with $n=6,7$ and $r$ is given as in Figure 1 .

Proof. We will give the proof in Section 6.2.

Remark 6.5. Suppose that $\mathfrak{g}$ is of type $\mathrm{A}_{n}$ and $\mathfrak{l}$ is of type $A_{r} \times A_{s}$ with $r+s=n-1$. The associated crystal $\mathbf{B}^{\mathrm{J}}$ can be realized as the set of $(r+1) \times(s+1)$ non-negative integral matrices (see [27, Section 4.3]). For $M \in \mathbf{B}^{\mathrm{J}}$, let $\lambda=\left(\lambda_{1}, \lambda_{2}, \ldots\right)$ be the shape of the tableaux corresponding to $M$ under RSK correspondence (cf. [10]). It was a well-known result due to Greene [11] that $\lambda_{1}$ is a maximal sum of entries in $M$ lying on a lattice path on $(r+1) \times(s+1)$ array of points from northeast to southwest, which coincides with $\varepsilon_{r}^{*}(M)$.

Similarly, for type $\mathrm{D}_{n}$ with $r=n$ (in this case, $\mathfrak{l}$ is of type $A_{n-1}$ ), the associated crystal $\mathbf{B}^{\mathrm{J}}$ can be realized as the set of the strictly upper triangular $n \times n$ nonnegative integral matrices (see [16, Section 3.2]). For $M \in \mathbf{B}^{\mathrm{J}}$, let $\lambda=\left(\lambda_{1}, \lambda_{2}, \ldots\right)$ be the shape of the tableaux corresponding to $M$ under Burge correspondence (see [3], cf. [16, Section 4.2]), which is an analog of RSK for type D. Then we proved in [16] that $\lambda_{1}$ is a maximal sum of entries in $M$ lying on a non-intersecting double path defined on $(n-1) \times(n-1)$ array of points (see [16, Definition 3.10] for definition of double path), which also coincides with $\varepsilon_{n}^{*}(M)$.

In this sense, we expect that there exists an analog of RSK for types $\mathrm{E}_{6}$ and $\mathrm{E}_{7}$ to explain the relationship between our model and a tableau model. Then, the formula in Theorem 6.4 may be viewed as a non-trivial type E analog of the previous results for $\varepsilon_{r}^{*}$.

Remark 6.6. By Theorem 6.4, we have

$$
\mathbf{B}^{\mathrm{J}, s}=\bigcap_{\mathbf{p}}\left\{\mathbf{c} \in \mathbf{B}^{\mathrm{J}} \mid\|\mathbf{c}\|_{\mathbf{p}} \leqslant s\right\},
$$

where $\mathbf{p}$ runs over the triple or quadruple paths in $\Delta$. Combining Theorem 5.1 , this gives a polytope realization of the $\mathrm{KR}$ crystal $B^{r, s}$ of types $\mathrm{E}_{6}^{(1)}$ and $\mathrm{E}_{7}^{(1)}$ with the minuscule nodes $r$ in Figure 1, which may be viewed as the type E analog of [16]. 
6.2. Proof of Theorem 6.4, We prove Theorem 6.4 following [16, Section 5.2]. We refer the reader to [16, Section 5.1] for the brief review of the formula of the transition matrix between Lusztig's parametrization and string parametrization of $B(\infty)$ [2], which is crucial to prove Theorem 6.4. We keep the notations in [16, Section 5.1].

6.2.1. A formula of $\varepsilon_{r}^{*}$. Let $\mathbf{i}_{0}$ be the reduced expression given in (3.2) with $\mathbf{i}^{\mathrm{J}}=$ $\left(i_{1}, \ldots, i_{M}\right)$ and $\mathbf{i}_{\mathrm{J}}=\left(i_{M+1}, \ldots, i_{N}\right)$, where $M$ and $N$ are given by

$$
(M, N)= \begin{cases}(16,36) & \text { if } \mathfrak{g} \text { is of type } \mathrm{E}_{6}, \\ (27,63) & \text { if } \mathfrak{g} \text { is of type } \mathrm{E}_{7} .\end{cases}
$$

If $\mathfrak{g}$ is of type $\mathrm{E}_{6}$, then $1^{*}=6,2^{*}=2,3^{*}=5,4^{*}=4,5^{*}=3,6^{*}=1$. If $\mathfrak{g}$ is of type $\mathrm{E}_{7}$, we have $i^{*}=i$ for all $i \in I$.

Put

$$
\mathbf{j}_{0}=\left(j_{1}, \ldots, j_{N}\right):=\mathbf{i}_{0}^{* o p}=\left(i_{N}^{*}, \ldots, i_{1}^{*}\right) .
$$

Remark 6.7. Since the representation $V\left(\varpi_{r}\right)$ of $U_{q}(\mathfrak{g})$ is minuscule, we can identify a $\mathbf{j}_{0}$-trail $\pi=\left(\nu_{0}, \ldots, \nu_{N}\right)$ in $V\left(\varpi_{n}\right)$ with a sequence $\left(b_{0}, \ldots, b_{N}\right) \in B\left(\varpi_{r}\right)^{\times(N+1)}$ such that $\operatorname{wt}\left(b_{k}\right)=\nu_{k}$ and $\tilde{f}_{j_{k}}^{d_{k}(\pi)} b_{k-1}=b_{k}$ with $d_{k}(\pi)=0$ or 1 for $1 \leqslant k \leqslant N$. Throughout this section, we frequently use this identification.

\section{Lemma 6.8.}

(1) There exists a unique $\mathbf{j}_{0}$-trail $\pi$ from $\varpi_{r}$ to $w_{0} \varpi_{r}$ such that $d_{k}(\pi)=0$ for $1 \leqslant k \leqslant N-M$, and $d_{k}(\pi)=1$ otherwise.

(2) There exists a unique $\left(j_{1}, \ldots, j_{N-M}\right)$-trail from $\varpi_{r}-\alpha_{r}$ to $\varpi_{r}-\theta$. We denote it by $\left(\tilde{\nu}_{0}, \ldots, \tilde{\nu}_{N-M}\right)$

Proof. The proof is straightforward by considering the structure of the crystal $B\left(\varpi_{r}\right)$ (cf. [18, Figures 2 and 3]) and $\mathbf{j}_{0}$ (6.1) under the identification in Remark 6.7.

Let $\mathcal{T}$ be the set of $\mathbf{j}_{0}$-trails from $s_{r} \varpi_{r}$ to $w_{0} \varpi_{r}$ in $V\left(\varpi_{r}\right)$. The following formula plays an important role in the proof of Theorem 6.4.

Lemma 6.9. For $\mathbf{c}=\left(c_{\beta_{k}}\right) \in \mathbf{B}^{\mathrm{J}}$, we have

$$
\varepsilon_{r}^{*}(\mathbf{c})=\max \left\{\|\mathbf{c}\|_{\pi} \mid \pi \in \mathcal{T}\right\},
$$

where $\|\mathbf{c}\|_{\pi}$ is defined by

$$
\|\mathbf{c}\|_{\pi}=\sum_{k=1}^{M}\left(1-d_{N-k+1}(\pi)\right) c_{\beta_{k}} .
$$


Proof. By using Lemma 6.8(1), the proof follows from the same argument in the one of [16, Lemma 5.4], where the formula of $\varepsilon_{r}^{*}(\mathbf{c})$ is obtained from the one of Berenstein-Zelevinsky [2] with respect to the reduced expressions $\mathbf{i}_{0}$ (3.2) and $\mathbf{j}_{0}$ (6.1).

Let us characterize the $\mathbf{j}_{0}$-trails $\pi \in \mathcal{T}$ such that $\|\mathbf{c}\|_{\pi}$ may be maximal for $\mathbf{c} \in \mathbf{B}^{\mathrm{J}}$. For $\pi=\left(\nu_{0}, \ldots, \nu_{N}\right) \in \mathcal{T}$, put $\pi_{\mathrm{J}}=\left(\nu_{0}, \ldots, \nu_{N-M}\right)$ and $\pi^{\mathrm{J}}=\left(\nu_{N-M+1}, \ldots, \nu_{N}\right)$. We define

$$
\mathcal{T}^{\prime}=\left\{\pi \in \mathcal{T} \mid \pi_{\mathrm{J}}=\left(\tilde{\nu}_{0}, \ldots, \tilde{\nu}_{N-M}\right)\right\},
$$

where $\left(\tilde{\nu}_{0}, \ldots, \tilde{\nu}_{N-M}\right)$ is the trail as in Lemma 6.8 $(2)$.

Lemma 6.10. For $\mathbf{c}=\left(c_{\beta_{k}}\right) \in \mathbf{B}^{\mathrm{J}}$, we have

$$
\varepsilon_{r}^{*}(\mathbf{c})=\max \left\{\|\mathbf{c}\|_{\pi} \mid \pi \in \mathcal{T}^{\prime}\right\} .
$$

Proof. Let $\pi=\left(\nu_{0}, \ldots, \nu_{N}\right) \in \mathcal{T} \backslash \mathcal{T}^{\prime}$ be given. It is enough to show that there exists $\pi^{\prime} \in \mathcal{T}^{\prime}$ such that $\|\mathbf{c}\|_{\pi} \leqslant\|\mathbf{c}\|_{\pi^{\prime}}$. To do this, let us consider the maximal subsequence $\left(j_{q_{1}}, \ldots, j_{q_{N^{\prime}}}\right)$ of $\left(j_{N-M+1}, \ldots, j_{N}\right)$ such that $k_{q_{k}}(\pi)=1$ for $1 \leqslant k \leqslant N^{\prime}$. Since $\pi \notin \mathcal{T}^{\prime}$, by considering the structure of the crystal $B\left(\varpi_{r}\right)$ (cf. [18, Figures 2 and 3]), the sequence $\left(j_{q_{1}}, \ldots, j_{q_{N^{\prime}}}\right)$ contains a subsequence $\left(j_{r_{1}}, \ldots, j_{r_{N^{\prime \prime}}}\right)$ given by

$$
\begin{cases}(1,3,4,5,6) & \text { for type } \mathrm{E}_{6} \text { with } r=1, \\ (6,5,4,3,1) & \text { for type } \mathrm{E}_{6} \text { with } r=6, \\ (7,6,5,4,3,2,4,5,6,7) & \text { for type } \mathrm{E}_{7} \text { with } r=7,\end{cases}
$$

(up to 2 -term braid moves on 2 and 3 in type $\mathrm{E}_{7}$ ). Then we define a $\mathbf{j}_{0}$-trail $\pi^{\prime}$ such that $\pi_{\mathrm{J}}^{\prime}=\left(\tilde{\nu}_{0}, \ldots, \tilde{\nu}_{N-M}\right)$ and

$$
d_{k}\left(\pi^{\prime}\right)= \begin{cases}1 & \text { if } k=r_{l} \text { for some } 1 \leqslant l \leqslant N^{\prime \prime} \\ 0 & \text { otherwise }\end{cases}
$$

Then $\pi^{\prime} \in \mathcal{T}^{\prime}$ and we have $\|\mathbf{c}\|_{\pi} \leqslant\|\mathbf{c}\|_{\pi^{\prime}}$ by (6.2) and the construction of $\pi^{\prime}$.

6.2.2. The $\mathbf{j}_{0}$-trails in $\mathcal{T}^{\prime}$. This subsection will be devoted to give the complete list of the $\mathbf{j}_{0}$-trails in $\mathcal{T}^{\prime}$. For $\pi \in \mathcal{T}^{\prime}$, since $\pi_{\mathrm{J}}$ is fixed by $\left(\tilde{\nu}_{0}, \ldots, \tilde{\nu}_{N-M}\right)$, we identify $\pi$ with the sequence $\left(d_{k}(\pi)\right)_{N-M+1 \leqslant k \leqslant N}$, where $d_{k}(\pi)$ is 0 or 1 (see Remark 6.7). For convenience, we also identify the sequence $\left(d_{k}(\pi)\right)_{N-M+1 \leqslant k \leqslant N}$ with a sequence given as in the following example.

Example 6.11. For type $\mathrm{E}_{6}$ with $r=6$, let $\pi \in \mathcal{T}^{\prime}$ be given. In this case, the subsequence $\left(\mathbf{i}^{\mathrm{J} *}\right)^{\text {op }}$ of $\mathbf{j}_{0}$ is given by

$$
(6,5,4,3,1,2,4,3,5,4,2,6,5,4,3,1)
$$


Then we rewrite the $k$-th number from left to right such that $d_{k}(\pi)=1$ (resp. $d_{k}(\pi)=0$ ) as the bold (resp. gray) one. If $\left(d_{k}(\pi)\right)_{21 \leqslant k \leqslant 36}$ for $\pi$ is given by $(1,0,0,0,0,0,0,0,1,1,0,0,0,0,1,1)$, then we identify it with

$$
(\mathbf{6}, 5,4,3,1,2,4,3, \mathbf{5}, \mathbf{4}, 2,6,5,4, \mathbf{3}, \mathbf{1}) .
$$

Remark 6.12. Let $\pi_{0}$ be the $\mathbf{j}_{0}$-trail in $\mathcal{T}^{\prime}$ corresponding to

$$
\begin{cases}(\mathbf{6}, \mathbf{5}, \mathbf{4}, \mathbf{3}, \mathbf{1}, 2,4,3,5,4,2,6,5,4,3,1) & \text { for type } \mathrm{E}_{6}, \\ (\mathbf{7}, \mathbf{6}, \mathbf{5}, \mathbf{4}, \mathbf{2}, \mathbf{3}, \mathbf{4}, \mathbf{5}, \mathbf{6}, \mathbf{7}, 1,3,4,5,6,2,4,5,3,4,2,1,3,4,5,6,7) & \text { for type } \mathrm{E}_{7},\end{cases}
$$

and let $\pi_{0}^{\prime}$ be the $\mathbf{j}_{0}$-trail in $\mathcal{T}^{\prime}$ corresponding to

$$
\begin{cases}(6,5,4,3,1,2,4,3,5,4,2, \mathbf{6}, \mathbf{5}, \mathbf{4}, \mathbf{3}, \mathbf{1}) & \text { for type } \mathrm{E}_{6}, \\ (7,6,5,4,2,3,4,5,6, \mathbf{7}, 1,3,4,5, \mathbf{6}, 2,4, \mathbf{5}, 3, \mathbf{4}, \mathbf{2}, 1, \mathbf{3}, \mathbf{4}, \mathbf{5}, \mathbf{6}, \mathbf{7}) & \text { for type } \mathrm{E}_{7} .\end{cases}
$$

Then any $\mathbf{j}_{0}$-trail $\pi$ in $\mathcal{T}^{\prime}$ is obtained from $\pi_{0}$ (resp. $\pi_{0}^{\prime}$ ) by moving the bold numbers to the right (resp. left) successively without a change of the enumeration on them (up to 2-term braid moves on 2 and 3 for type $\mathrm{E}_{7}$ ).

Now, we state the complete list of the $\mathbf{j}_{0}$-trails in $\mathcal{T}^{\prime}$ as follows.

Case 1. Type $\mathrm{E}_{6}$. We consider the case of $r=6$ only, since the case of $r=1$ is almost identical.

$$
\begin{aligned}
& (\mathbf{6}, \mathbf{5}, \mathbf{4}, \mathbf{3}, \mathbf{1}, 2,4,3,5,4,2,6,5,4,3,1), \\
& (\mathbf{6}, \mathbf{5}, \mathbf{4}, \mathbf{3}, 1,2,4,3,5,4,2,6,5,4,3, \mathbf{1}), \\
& (\mathbf{6}, \mathbf{5}, \mathbf{4}, 3,1,2,4, \mathbf{3}, 5,4,2,6,5,4,3, \mathbf{1}), \\
& (\mathbf{6}, \mathbf{5}, 4,3,1,2, \mathbf{4}, \mathbf{3}, 5,4,2,6,5,4,3, \mathbf{1}), \\
& (\mathbf{6}, \mathbf{5}, \mathbf{4}, 3,1,2,4,3,5,4,2,6,5,4, \mathbf{3}, \mathbf{1}), \\
& (\mathbf{6}, \mathbf{5}, 4,3,1,2, \mathbf{4}, 3,5,4,2,6,5,4, \mathbf{3}, \mathbf{1}), \\
& (\mathbf{6}, \mathbf{5}, 4,3,1,2,4,3,5, \mathbf{4}, 2,6,5,4, \mathbf{3}, \mathbf{1}), \\
& (\mathbf{6}, 5,4,3,1,2,4,3, \mathbf{5}, \mathbf{4}, 2,6,5,4, \mathbf{3}, \mathbf{1}), \\
& (\mathbf{6}, \mathbf{5}, 4,3,1,2,4,3,5,4,2,6,5, \mathbf{4}, \mathbf{3}, \mathbf{1}), \\
& (\mathbf{6}, 5,4,3,1,2,4,3, \mathbf{5}, 4,2,6,5, \mathbf{4}, \mathbf{3}, \mathbf{1}), \\
& (\mathbf{6}, 5,4,3,1,2,4,3,5,4,2,6, \mathbf{5}, \mathbf{4}, \mathbf{3}, \mathbf{1}), \\
& (6,5,4,3,1,2,4,3,5,4,2, \mathbf{6}, \mathbf{5}, \mathbf{4}, \mathbf{3}, \mathbf{1}) .
\end{aligned}
$$

Case 2. Type $\mathrm{E}_{7}$. In this case, since the subsequence (6.3) involves both 2 and 3 which commutes, we separate two cases along the position of them.

Sub-Case 1. (7, 6, 5, 4, 2, 3, 4, 5, 6, 7). 
$(\mathbf{7}, \mathbf{6}, \mathbf{5}, \mathbf{4}, \mathbf{2}, \mathbf{3}, \mathbf{4}, \mathbf{5}, \mathbf{6}, 7,1,3,4,5,6,2,4,5,3,4,2,1,3,4,5,6, \mathbf{7})$, $(\mathbf{7}, \mathbf{6}, \mathbf{5}, \mathbf{4}, \mathbf{2}, \mathbf{3}, \mathbf{4}, \mathbf{5}, 6,7,1,3,4,5, \mathbf{6}, 2,4,5,3,4,2,1,3,4,5,6, \mathbf{7})$, $(\mathbf{7}, \mathbf{6}, \mathbf{5}, \mathbf{4}, \mathbf{2}, \mathbf{3}, \mathbf{4}, 5,6,7,1,3,4, \mathbf{5}, \mathbf{6}, 2,4,5,3,4,2,1,3,4,5,6, \mathbf{7})$, $(\mathbf{7}, \mathbf{6}, \mathbf{5}, \mathbf{4}, \mathbf{2}, \mathbf{3}, 4,5,6,7,1,3, \mathbf{4}, \mathbf{5}, \mathbf{6}, 2,4,5,3,4,2,1,3,4,5,6, \mathbf{7})$, $(\mathbf{7}, \mathbf{6}, \mathbf{5}, \mathbf{4}, \mathbf{2}, 3,4,5,6,7,1, \mathbf{3}, \mathbf{4}, \mathbf{5}, \mathbf{6}, 2,4,5,3,4,2,1,3,4,5,6, \mathbf{7})$, $(\mathbf{7}, \mathbf{6}, \mathbf{5}, \mathbf{4}, \mathbf{2}, \mathbf{3}, \mathbf{4}, \mathbf{5}, 6,7,1,3,4,5,6,2,4,5,3,4,2,1,3,4,5, \mathbf{6}, \mathbf{7})$, $(\mathbf{7}, \mathbf{6}, \mathbf{5}, \mathbf{4}, \mathbf{2}, \mathbf{3}, \mathbf{4}, 5,6,7,1,3,4, \mathbf{5}, 6,2,4,5,3,4,2,1,3,4,5, \mathbf{6}, \mathbf{7})$, $(\mathbf{7}, \mathbf{6}, \mathbf{5}, \mathbf{4}, \mathbf{2}, \mathbf{3}, 4,5,6,7,1,3, \mathbf{4}, \mathbf{5}, 6,2,4,5,3,4,2,1,3,4,5, \mathbf{6}, \mathbf{7})$, $(\mathbf{7}, \mathbf{6}, \mathbf{5}, \mathbf{4}, \mathbf{2}, 3,4,5,6,7,1, \mathbf{3}, \mathbf{4}, \mathbf{5}, 6,2,4,5,3,4,2,1,3,4,5, \mathbf{6}, \mathbf{7})$, $(\mathbf{7}, \mathbf{6}, \mathbf{5}, \mathbf{4}, \mathbf{2}, \mathbf{3}, \mathbf{4}, 5,6,7,1,3,4,5,6,2,4, \mathbf{5}, 3,4,2,1,3,4,5, \mathbf{6}, \mathbf{7})$, $(\mathbf{7}, \mathbf{6}, \mathbf{5}, \mathbf{4}, \mathbf{2}, \mathbf{3}, 4,5,6,7,1,3, \mathbf{4}, 5,6,2,4, \mathbf{5}, 3,4,2,1,3,4,5, \mathbf{6}, \mathbf{7})$, $(\mathbf{7}, \mathbf{6}, \mathbf{5}, \mathbf{4}, \mathbf{2}, 3,4,5,6,7,1, \mathbf{3}, \mathbf{4}, 5,6,2,4, \mathbf{5}, 3,4,2,1,3,4,5, \mathbf{6}, \mathbf{7})$, $(\mathbf{7}, \mathbf{6}, \mathbf{5}, \mathbf{4}, \mathbf{2}, \mathbf{3}, 4,5,6,7,1,3,4,5,6,2, \mathbf{4}, \mathbf{5}, 3,4,2,1,3,4,5, \mathbf{6}, \mathbf{7})$, $(\mathbf{7}, \mathbf{6}, \mathbf{5}, \mathbf{4}, \mathbf{2}, 3,4,5,6,7,1, \mathbf{3}, 4,5,6,2, \mathbf{4}, \mathbf{5}, 3,4,2,1,3,4,5, \mathbf{6}, \mathbf{7})$, $(\mathbf{7}, \mathbf{6}, \mathbf{5}, \mathbf{4}, \mathbf{2}, \mathbf{3}, \mathbf{4}, 5,6,7,1,3,4,5,6,2,4,5,3,4,2,1,3,4, \mathbf{5}, \mathbf{6}, \mathbf{7})$, $(\mathbf{7}, \mathbf{6}, \mathbf{5}, \mathbf{4}, \mathbf{2}, \mathbf{3}, 4,5,6,7,1,3, \mathbf{4}, 5,6,2,4,5,3,4,2,1,3,4, \mathbf{5}, \mathbf{6}, \mathbf{7})$, $(\mathbf{7}, \mathbf{6}, \mathbf{5}, \mathbf{4}, \mathbf{2}, 3,4,5,6,7,1, \mathbf{3}, \mathbf{4}, 5,6,2,4,5,3,4,2,1,3,4, \mathbf{5}, \mathbf{6}, \mathbf{7})$, $(\mathbf{7}, \mathbf{6}, \mathbf{5}, \mathbf{4}, \mathbf{2}, \mathbf{3}, 4,5,6,7,1,3,4,5,6,2, \mathbf{4}, 5,3,4,2,1,3,4, \mathbf{5}, \mathbf{6}, \mathbf{7})$, $(\mathbf{7}, \mathbf{6}, \mathbf{5}, \mathbf{4}, \mathbf{2}, \mathbf{3}, 4,5,6,7,1,3,4,5,6,2,4,5,3, \mathbf{4}, 2,1,3,4, \mathbf{5}, \mathbf{6}, \mathbf{7})$ $(\mathbf{7}, \mathbf{6}, \mathbf{5}, \mathbf{4}, \mathbf{2}, 3,4,5,6,7,1, \mathbf{3}, 4,5,6,2,4,5,3, \mathbf{4}, 2,1,3,4, \mathbf{5}, \mathbf{6}, \mathbf{7})$ $(\mathbf{7}, \mathbf{6}, \mathbf{5}, \mathbf{4}, \mathbf{2}, 3,4,5,6,7,1, \mathbf{3}, 4,5,6,2, \mathbf{4}, 5,3,4,2,1,3,4, \mathbf{5}, \mathbf{6}, \mathbf{7})$, $(\mathbf{7}, \mathbf{6}, \mathbf{5}, \mathbf{4}, \mathbf{2}, \mathbf{3}, 4,5,6,7,1,3,4,5,6,2,4,5,3,4,2,1,3, \mathbf{4}, \mathbf{5}, \mathbf{6}, \mathbf{7})$, $(\mathbf{7}, \mathbf{6}, \mathbf{5}, \mathbf{4}, \mathbf{2}, 3,4,5,6,7,1, \mathbf{3}, 4,5,6,2,4,5,3,4,2,1,3, \mathbf{4}, \mathbf{5}, \mathbf{6}, \mathbf{7})$, $(\mathbf{7}, \mathbf{6}, \mathbf{5}, \mathbf{4}, \mathbf{2}, 3,4,5,6,7,1,3,4,5,6,2,4,5, \mathbf{3}, 4,2,1,3, \mathbf{4}, \mathbf{5}, \mathbf{6}, \mathbf{7})$, $(\mathbf{7}, \mathbf{6}, \mathbf{5}, \mathbf{4}, 2,3,4,5,6,7,1,3,4,5,6, \mathbf{2}, 4,5, \mathbf{3}, 4,2,1,3, \mathbf{4}, \mathbf{5}, \mathbf{6}, \mathbf{7})$, $(\mathbf{7}, \mathbf{6}, \mathbf{5}, 4,2,3, \mathbf{4}, 5,6,7,1,3,4,5,6, \mathbf{2}, 4,5, \mathbf{3}, 4,2,1,3, \mathbf{4}, \mathbf{5}, \mathbf{6}, \mathbf{7})$, $(\mathbf{7}, \mathbf{6}, \mathbf{5}, 4,2,3,4,5,6,7,1,3, \mathbf{4}, 5,6, \mathbf{2}, 4,5, \mathbf{3}, 4,2,1,3, \mathbf{4}, \mathbf{5}, \mathbf{6}, \mathbf{7})$, $(\mathbf{7}, \mathbf{6}, 5,4,2,3,4, \mathbf{5}, 6,7,1,3, \mathbf{4}, 5,6, \mathbf{2}, 4,5, \mathbf{3}, 4,2,1,3, \mathbf{4}, \mathbf{5}, \mathbf{6}, \mathbf{7})$, $(\mathbf{7}, \mathbf{6}, \mathbf{5}, \mathbf{4}, 2,3,4,5,6,7,1,3,4,5,6, \mathbf{2}, 4,5, \mathbf{3}, \mathbf{4}, 2,1,3,4, \mathbf{5}, \mathbf{6}, \mathbf{7})$, $(\mathbf{7}, \mathbf{6}, \mathbf{5}, 4,2,3, \mathbf{4}, 5,6,7,1,3,4,5,6, \mathbf{2}, 4,5, \mathbf{3}, \mathbf{4}, 2,1,3,4, \mathbf{5}, \mathbf{6}, \mathbf{7})$, $(\mathbf{7}, \mathbf{6}, \mathbf{5}, 4,2,3,4,5,6,7,1,3, \mathbf{4}, 5,6, \mathbf{2}, 4,5, \mathbf{3}, \mathbf{4}, 2,1,3,4, \mathbf{5}, \mathbf{6}, \mathbf{7})$, $(\mathbf{7}, \mathbf{6}, \mathbf{5}, \mathbf{4}, \mathbf{2}, 3,4,5,6,7,1,3,4,5,6,2,4,5,3,4,2,1, \mathbf{3}, \mathbf{4}, \mathbf{5}, \mathbf{6}, \mathbf{7})$, $(\mathbf{7}, \mathbf{6}, \mathbf{5}, \mathbf{4}, 2,3,4,5,6,7,1,3,4,5,6, \mathbf{2}, 4,5,3,4,2,1, \mathbf{3}, \mathbf{4}, \mathbf{5}, \mathbf{6}, \mathbf{7})$, $(\mathbf{7}, \mathbf{6}, \mathbf{5}, 4,2,3, \mathbf{4}, 5,6,7,1,3,4,5,6, \mathbf{2}, 4,5,3,4,2,1, \mathbf{3}, \mathbf{4}, \mathbf{5}, \mathbf{6}, \mathbf{7})$, $(\mathbf{7}, \mathbf{6}, \mathbf{5}, 4,2,3,4,5,6,7,1,3, \mathbf{4}, 5,6, \mathbf{2}, 4,5,3,4,2,1, \mathbf{3}, \mathbf{4}, \mathbf{5}, \mathbf{6}, \mathbf{7})$, $(\mathbf{7}, \mathbf{6}, 5,4,2,3,4, \mathbf{5}, 6,7,1,3, \mathbf{4}, 5,6, \mathbf{2}, 4,5,3,4,2,1, \mathbf{3}, \mathbf{4}, \mathbf{5}, \mathbf{6}, \mathbf{7})$, 
$(\mathbf{7}, \mathbf{6}, \mathbf{5}, \mathbf{4}, 2,3,4,5,6,7,1,3,4,5,6,2,4,5,3,4, \mathbf{2}, 1, \mathbf{3}, \mathbf{4}, \mathbf{5}, \mathbf{6}, \mathbf{7})$,

$(\mathbf{7}, \mathbf{6}, \mathbf{5}, 4,2,3, \mathbf{4}, 5,6,7,1,3,4,5,6,2,4,5,3,4, \mathbf{2}, 1, \mathbf{3}, \mathbf{4}, \mathbf{5}, \mathbf{6}, \mathbf{7})$,

$(\mathbf{7}, \mathbf{6}, \mathbf{5}, 4,2,3,4,5,6,7,1,3, \mathbf{4}, 5,6,2,4,5,3,4, \mathbf{2}, 1, \mathbf{3}, \mathbf{4}, \mathbf{5}, \mathbf{6}, \mathbf{7})$,

$(\mathbf{7}, \mathbf{6}, 5,4,2,3,4, \mathbf{5}, 6,7,1,3, \mathbf{4}, 5,6,2,4,5,3,4, \mathbf{2}, 1, \mathbf{3}, \mathbf{4}, \mathbf{5}, \mathbf{6}, \mathbf{7})$,

$(\mathbf{7}, \mathbf{6}, \mathbf{5}, 4,2,3,4,5,6,7,1,3,4,5,6,2, \mathbf{4}, 5,3,4, \mathbf{2}, 1, \mathbf{3}, \mathbf{4}, \mathbf{5}, \mathbf{6}, \mathbf{7})$,

$(\mathbf{7}, \mathbf{6}, 5,4,2,3,4, \mathbf{5}, 6,7,1,3,4,5,6,2, \mathbf{4}, 5,3,4, \mathbf{2}, 1, \mathbf{3}, \mathbf{4}, \mathbf{5}, \mathbf{6}, \mathbf{7})$,

$(\mathbf{7}, \mathbf{6}, 5,4,2,3,4,5,6,7,1,3,4, \mathbf{5}, 6,2, \mathbf{4}, 5,3,4, \mathbf{2}, 1, \mathbf{3}, \mathbf{4}, \mathbf{5}, \mathbf{6}, \mathbf{7})$,

$(\mathbf{7}, 6,5,4,2,3,4,5, \mathbf{6}, 7,1,3,4, \mathbf{5}, 6,2, \mathbf{4}, 5,3,4, \mathbf{2}, 1, \mathbf{3}, \mathbf{4}, \mathbf{5}, \mathbf{6}, \mathbf{7})$,

$(\mathbf{7}, \mathbf{6}, \mathbf{5}, 4,2,3,4,5,6,7,1,3,4,5,6,2,4,5,3, \mathbf{4}, \mathbf{2}, 1, \mathbf{3}, \mathbf{4}, \mathbf{5}, \mathbf{6}, \mathbf{7})$,

$(\mathbf{7}, \mathbf{6}, 5,4,2,3,4, \mathbf{5}, 6,7,1,3,4,5,6,2,4,5,3, \mathbf{4}, \mathbf{2}, 1, \mathbf{3}, \mathbf{4}, \mathbf{5}, \mathbf{6}, \mathbf{7})$,

$(\mathbf{7}, \mathbf{6}, 5,4,2,3,4,5,6,7,1,3,4, \mathbf{5}, 6,2,4,5,3, \mathbf{4}, \mathbf{2}, 1, \mathbf{3}, \mathbf{4}, \mathbf{5}, \mathbf{6}, \mathbf{7})$,

$(\mathbf{7}, 6,5,4,2,3,4,5, \mathbf{6}, 7,1,3,4, \mathbf{5}, 6,2,4,5,3, \mathbf{4}, \mathbf{2}, 1, \mathbf{3}, \mathbf{4}, \mathbf{5}, \mathbf{6}, \mathbf{7})$,

$(\mathbf{7}, \mathbf{6}, 5,4,2,3,4,5,6,7,1,3,4,5,6,2,4, \mathbf{5}, 3, \mathbf{4}, \mathbf{2}, 1, \mathbf{3}, \mathbf{4}, \mathbf{5}, \mathbf{6}, \mathbf{7})$,

$(\mathbf{7}, 6,5,4,2,3,4,5, \mathbf{6}, 7,1,3,4,5,6,2,4, \mathbf{5}, 3, \mathbf{4}, \mathbf{2}, 1, \mathbf{3}, \mathbf{4}, \mathbf{5}, \mathbf{6}, \mathbf{7})$,

$(\mathbf{7}, 6,5,4,2,3,4,5,6,7,1,3,4,5, \mathbf{6}, 2,4, \mathbf{5}, 3, \mathbf{4}, \mathbf{2}, 1, \mathbf{3}, \mathbf{4}, \mathbf{5}, \mathbf{6}, \mathbf{7})$,

$(7,6,5,4,2,3,4,5,6, \mathbf{7}, 1,3,4,5, \mathbf{6}, 2,4, \mathbf{5}, 3, \mathbf{4}, \mathbf{2}, 1, \mathbf{3}, \mathbf{4}, \mathbf{5}, \mathbf{6}, \mathbf{7})$.

Sub-Case 2. $(7,6,5,4, \mathbf{3}, \mathbf{2}, 4,5,6,7)$.

$(\mathbf{7}, \mathbf{6}, \mathbf{5}, \mathbf{4}, 2, \mathbf{3}, 4,5,6,7,1,3,4,5,6, \mathbf{2}, \mathbf{4}, \mathbf{5}, 3,4,2,1,3,4,5, \mathbf{6}, \mathbf{7})$,

$(\mathbf{7}, \mathbf{6}, \mathbf{5}, \mathbf{4}, 2,3,4,5,6,7,1, \mathbf{3}, 4,5,6, \mathbf{2}, \mathbf{4}, \mathbf{5}, 3,4,2,1,3,4,5, \mathbf{6}, \mathbf{7})$,

$(\mathbf{7}, \mathbf{6}, \mathbf{5}, 4,2,3, \mathbf{4}, 5,6,7,1, \mathbf{3}, 4,5,6, \mathbf{2}, \mathbf{4}, \mathbf{5}, 3,4,2,1,3,4,5, \mathbf{6}, \mathbf{7})$,

$(\mathbf{7}, \mathbf{6}, \mathbf{5}, \mathbf{4}, 2, \mathbf{3}, 4,5,6,7,1,3,4,5,6, \mathbf{2}, \mathbf{4}, 5,3,4,2,1,3,4, \mathbf{5}, \mathbf{6}, \mathbf{7})$,

$(\mathbf{7}, \mathbf{6}, \mathbf{5}, \mathbf{4}, 2,3,4,5,6,7,1, \mathbf{3}, 4,5,6, \mathbf{2}, \mathbf{4}, 5,3,4,2,1,3,4, \mathbf{5}, \mathbf{6}, \mathbf{7})$,

$(\mathbf{7}, \mathbf{6}, \mathbf{5}, 4,2,3, \mathbf{4}, 5,6,7,1, \mathbf{3}, 4,5,6, \mathbf{2}, \mathbf{4}, 5,3,4,2,1,3,4, \mathbf{5}, \mathbf{6}, \mathbf{7})$,

$(\mathbf{7}, \mathbf{6}, \mathbf{5}, \mathbf{4}, 2, \mathbf{3}, 4,5,6,7,1,3,4,5,6, \mathbf{2}, 4,5,3, \mathbf{4}, 2,1,3,4, \mathbf{5}, \mathbf{6}, \mathbf{7})$,

$(\mathbf{7}, \mathbf{6}, \mathbf{5}, \mathbf{4}, 2,3,4,5,6,7,1, \mathbf{3}, 4,5,6, \mathbf{2}, 4,5,3, \mathbf{4}, 2,1,3,4, \mathbf{5}, \mathbf{6}, \mathbf{7})$,

$(\mathbf{7}, \mathbf{6}, \mathbf{5}, 4,2,3, \mathbf{4}, 5,6,7,1, \mathbf{3}, 4,5,6, \mathbf{2}, 4,5,3, \mathbf{4}, 2,1,3,4, \mathbf{5}, \mathbf{6}, \mathbf{7})$,

$(\mathbf{7}, \mathbf{6}, \mathbf{5}, \mathbf{4}, 2, \mathbf{3}, 4,5,6,7,1,3,4,5,6, \mathbf{2}, 4,5,3,4,2,1,3, \mathbf{4}, \mathbf{5}, \mathbf{6}, \mathbf{7})$,

$(\mathbf{7}, \mathbf{6}, \mathbf{5}, \mathbf{4}, 2, \mathbf{3}, 4,5,6,7,1,3,4,5,6,2,4,5,3,4, \mathbf{2}, 1,3, \mathbf{4}, \mathbf{5}, \mathbf{6}, \mathbf{7})$,

$(\mathbf{7}, \mathbf{6}, \mathbf{5}, \mathbf{4}, 2,3,4,5,6,7,1, \mathbf{3}, 4,5,6,2,4,5,3,4, \mathbf{2}, 1,3, \mathbf{4}, \mathbf{5}, \mathbf{6}, \mathbf{7})$,

$(\mathbf{7}, \mathbf{6}, \mathbf{5}, 4,2,3, \mathbf{4}, 5,6,7,1, \mathbf{3}, 4,5,6,2,4,5,3,4, \mathbf{2}, 1,3, \mathbf{4}, \mathbf{5}, \mathbf{6}, \mathbf{7})$,

$(\mathbf{7}, \mathbf{6}, \mathbf{5}, \mathbf{4}, 2,3,4,5,6,7,1,3,4,5,6,2,4,5, \mathbf{3}, 4, \mathbf{2}, 1,3, \mathbf{4}, \mathbf{5}, \mathbf{6}, \mathbf{7})$,

$(\mathbf{7}, \mathbf{6}, \mathbf{5}, 4,2,3, \mathbf{4}, 5,6,7,1,3,4,5,6,2,4,5, \mathbf{3}, 4, \mathbf{2}, 1,3, \mathbf{4}, \mathbf{5}, \mathbf{6}, \mathbf{7})$,

$(\mathbf{7}, \mathbf{6}, \mathbf{5}, 4,2,3,4,5,6,7,1,3, \mathbf{4}, 5,6,2,4,5, \mathbf{3}, 4, \mathbf{2}, 1,3, \mathbf{4}, \mathbf{5}, \mathbf{6}, \mathbf{7})$,

$(\mathbf{7}, \mathbf{6}, 5,4,2,3,4, \mathbf{5}, 6,7,1,3, \mathbf{4}, 5,6,2,4,5, \mathbf{3}, 4, \mathbf{2}, 1,3, \mathbf{4}, \mathbf{5}, \mathbf{6}, \mathbf{7})$,

$(\mathbf{7}, \mathbf{6}, \mathbf{5}, 4,2,3,4,5,6,7,1,3,4,5,6,2, \mathbf{4}, 5, \mathbf{3}, 4, \mathbf{2}, 1,3, \mathbf{4}, \mathbf{5}, \mathbf{6}, \mathbf{7})$, 


$$
\begin{aligned}
& (\mathbf{7}, \mathbf{6}, 5,4,2,3,4, \mathbf{5}, 6,7,1,3,4,5,6,2, \mathbf{4}, 5, \mathbf{3}, 4, \mathbf{2}, 1,3, \mathbf{4}, \mathbf{5}, \mathbf{6}, \mathbf{7}), \\
& (\mathbf{7}, \mathbf{6}, 5,4,2,3,4,5,6,7,1,3,4, \mathbf{5}, 6,2, \mathbf{4}, 5, \mathbf{3}, 4, \mathbf{2}, 1,3, \mathbf{4}, \mathbf{5}, \mathbf{6}, \mathbf{7}), \\
& (\mathbf{7}, 6,5,4,2,3,4,5, \mathbf{6}, 7,1,3,4, \mathbf{5}, 6,2, \mathbf{4}, 5, \mathbf{3}, 4, \mathbf{2}, 1,3, \mathbf{4}, \mathbf{5}, \mathbf{6}, \mathbf{7}), \\
& (\mathbf{7}, \mathbf{6}, \mathbf{5}, \mathbf{4}, 2,3,4,5,6,7,1, \mathbf{3}, 4,5,6, \mathbf{2}, 4,5,3,4,2,1,3, \mathbf{4}, \mathbf{5}, \mathbf{6}, \mathbf{7}), \\
& (\mathbf{7}, \mathbf{6}, \mathbf{5}, 4,2,3, \mathbf{4}, 5,6,7,1, \mathbf{3}, 4,5,6, \mathbf{2}, 4,5,3,4,2,1,3, \mathbf{4}, \mathbf{5}, \mathbf{6}, \mathbf{7}) .
\end{aligned}
$$

Remark 6.13. The configurations of the paths in Definition 6.2 are devised following the above description of the $\mathbf{j}_{0}$-trails in $\mathcal{T}^{\prime}$ (cf. Examples 6.14 and 6.16).

6.2.3. Description of $\mathbf{j}_{0}$-trails in $\mathcal{T}^{\prime}$. In this subsection, we describe the $\mathbf{j}_{0}$-trails in $\mathcal{T}^{\prime}$ in terms of the path on $\Delta$. From now on, we assume $r=n$ for simplicity.

Let $\mathcal{D}$ be the set of arrays, where either 0 or 1 is placed in each row of $\Delta^{\mathrm{J}}$. For $\pi \in$ $\mathcal{T}^{\prime}$, we can identify a $\mathbf{j}_{0}$-trail $\pi$ in $\mathcal{T}^{\prime}$ with a unique array $\mathbf{d}(\pi)=\left(d_{k}(\pi)\right)_{N-M+1 \leqslant k \leqslant N}$ of $\mathcal{D}$ determined as follows.

Case 1. Type $\mathrm{E}_{6}$. In this case, the array $\mathbf{d}(\pi)$ is given by

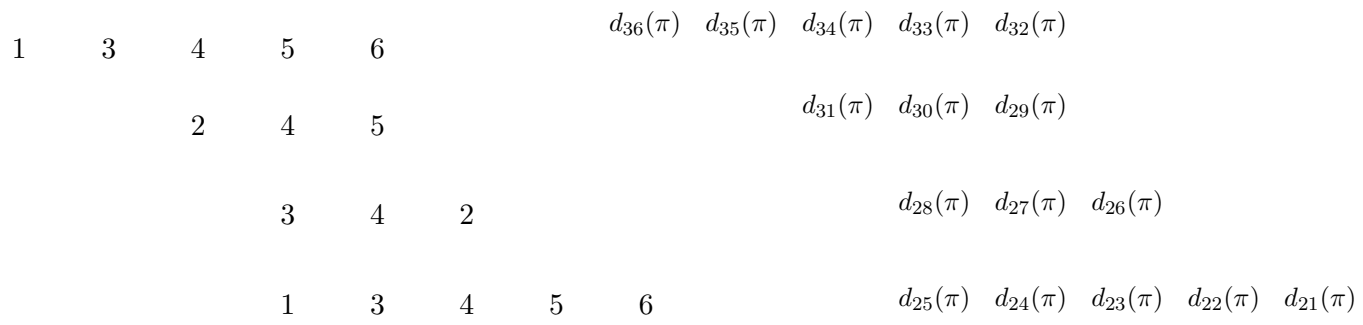

Case 2. Type $\mathrm{E}_{7}$. In this case, the array $\mathbf{d}(\pi)$ is given by

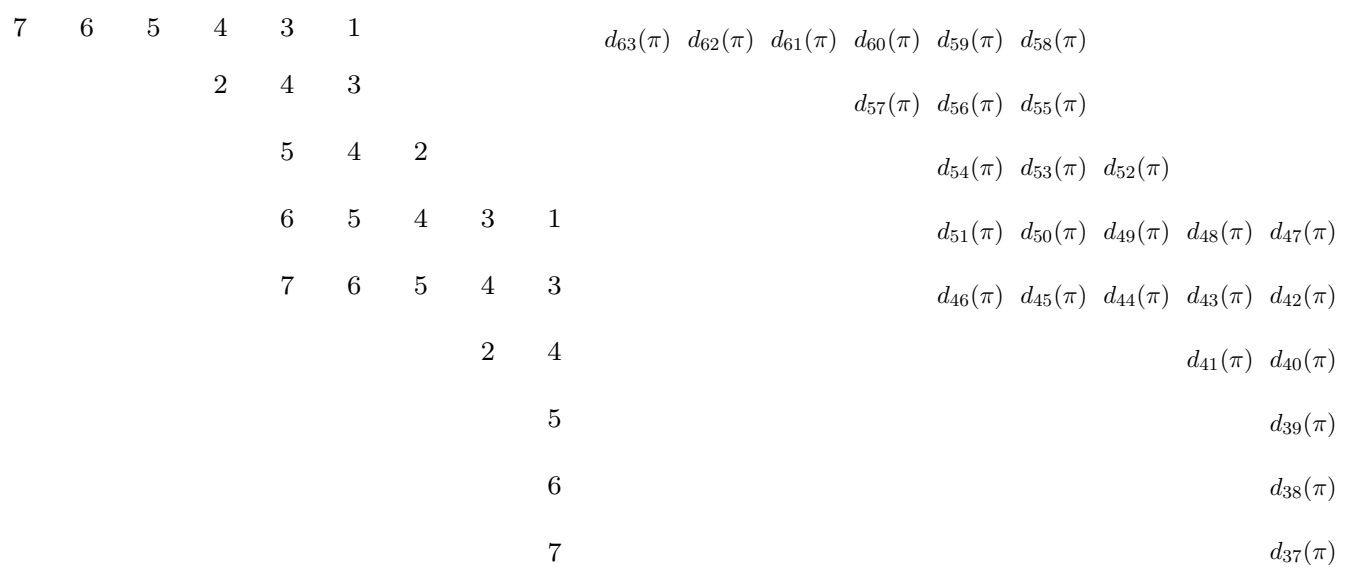


Then it is obvious that the map

$$
\begin{aligned}
& \mathcal{T}^{\prime} \longrightarrow \mathcal{D} \\
& \pi \longmapsto \mathbf{d}(\pi)
\end{aligned}
$$

is injective since $\pi_{\mathrm{J}}$ is fixed by $\left(\tilde{\nu}_{0}, \ldots, \tilde{\nu}_{N-M}\right)$. Let $\mathcal{D}^{\mathrm{J}}$ be the image of $\mathcal{T}^{\prime}$ under the above map. Thanks to Section 6.2.2, we know the complete description of the image $\mathcal{D}^{\mathrm{J}}$.

Example 6.14. Let us consider the case of type $E_{7}$. Let $\pi \in \mathcal{T}^{\prime}$ be the $\mathbf{j}_{0}$-trail corresponding to

$$
(\mathbf{7}, \mathbf{6}, \mathbf{5}, 4,2,3, \mathbf{4}, 5,6,7,1,3,4,5,6, \mathbf{2}, 4,5, \mathbf{3}, 4,2,1,3, \mathbf{4}, \mathbf{5}, \mathbf{6}, \mathbf{7}) .
$$

Then $\mathbf{d}(\pi)$ is given by

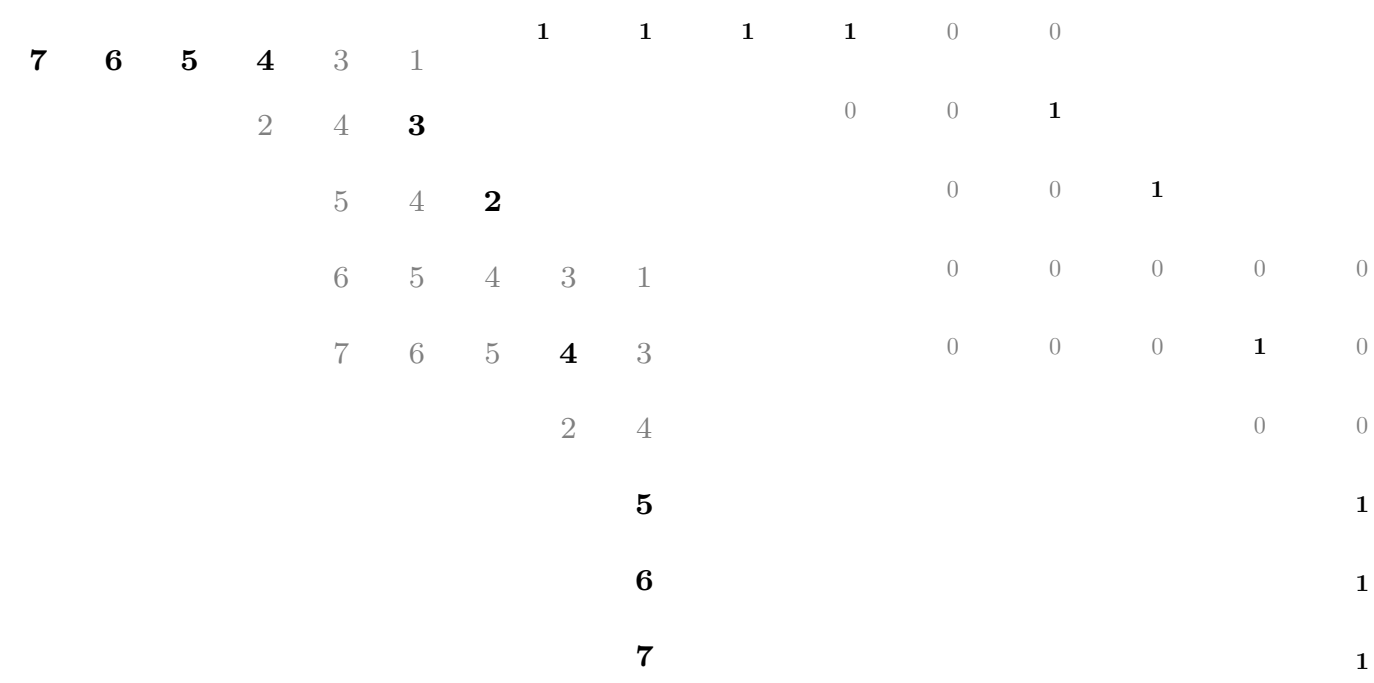

By Remark 6.12, for any $\pi \in \mathcal{T}^{\prime}$, there exists a sequence $\pi_{1}, \ldots, \pi_{\ell}$ of $\mathcal{T}^{\prime}$ such that $\pi_{\ell}=\pi$ and $\pi_{k+1}$ is obtained from $\pi_{k}$ by moving a bold number to the right for $0 \leqslant k \leqslant \ell-1$. Let $i_{k}$ be the bold number moved in $\pi_{k}$. Then under the identification via the map (6.4), the array $\mathbf{d}\left(\pi_{k+1}\right)$ is obtained from $\mathbf{d}\left(\pi_{k}\right)$ by moving 1 on $\mathbf{d}\left(\pi_{k}\right)$ located at the position of $i_{k}$ along the dashed arrow between $i_{k}$ 's in (6.5). 


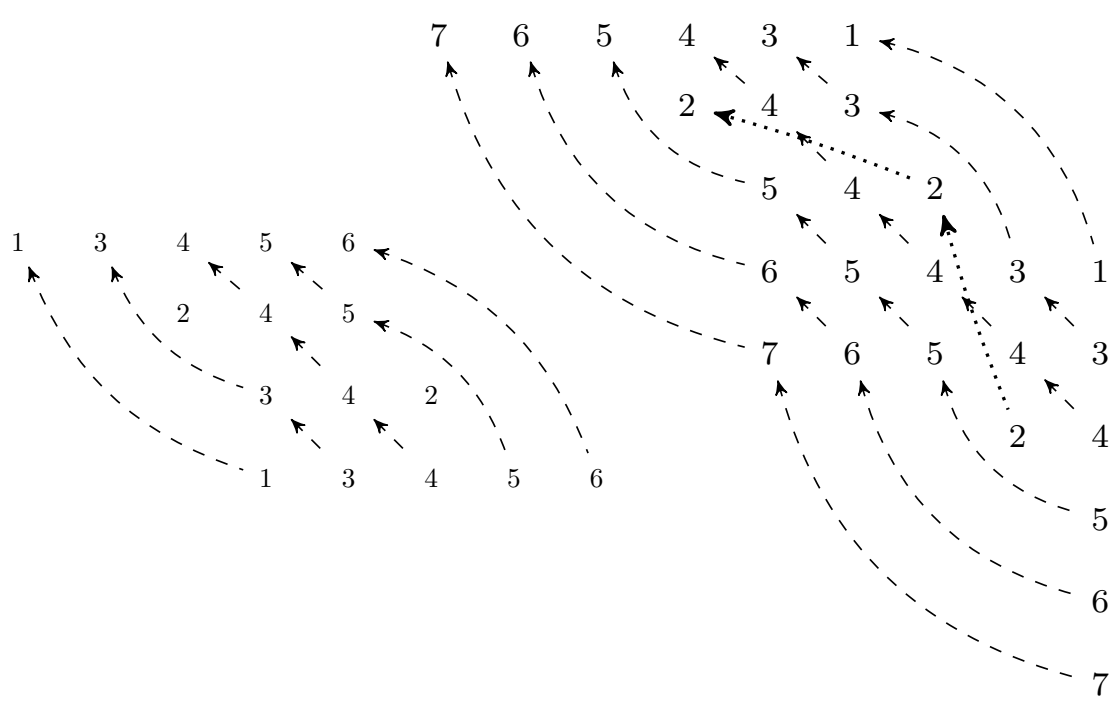

Example 6.15. Let $\pi$ be the $\mathbf{j}_{0}$-trail in Example 6.14 and let $\pi^{\prime}$ be the $\mathbf{j}_{0}$-trail corresponding to the following sequence

$$
(\mathbf{7}, \mathbf{6}, \mathbf{5}, 4,2,3, \mathbf{4}, 5,6,7,1,3,4,5,6,2,4,5, \mathbf{3}, 4, \mathbf{2}, 1,3, \mathbf{4}, \mathbf{5}, \mathbf{6}, \mathbf{7}),
$$

which is obtained from $\pi$ by moving 2 to the right. Then we have

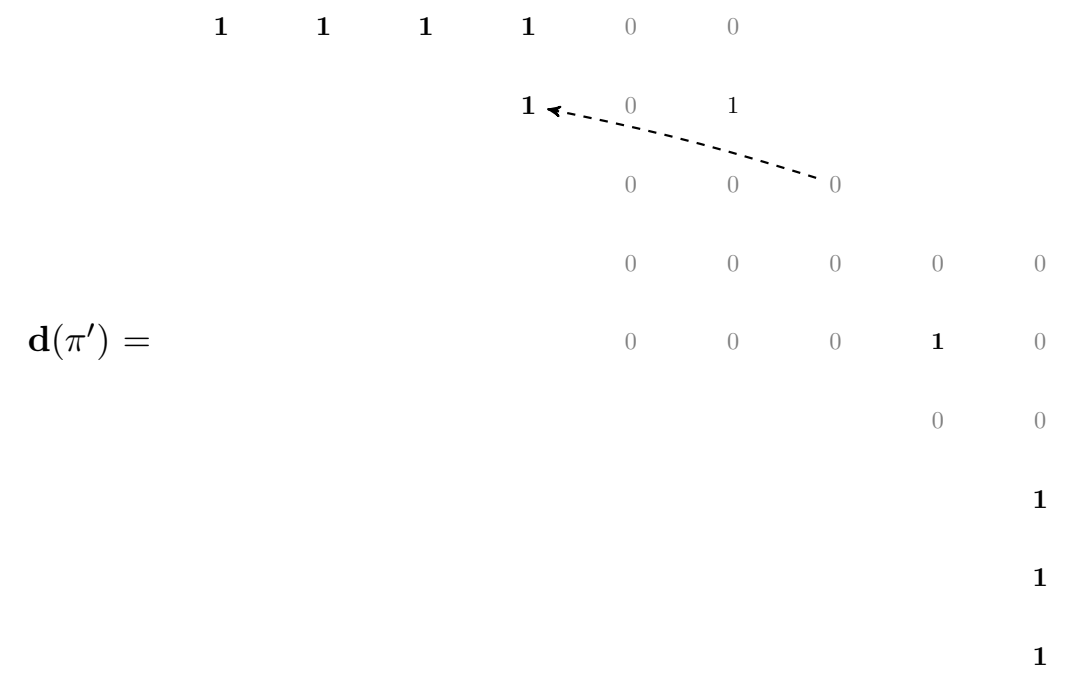

Under the enumeration of $d_{k}(\pi)$ on $\mathbf{d}(\pi)$, for $\mathbf{p} \in \mathcal{P}_{n}$, we define $\mathbf{d}(\mathbf{p})=\left(d_{k}\right) \in \mathcal{D}$ by

$$
d_{k}= \begin{cases}0 & \mathbf{p} \cap \Delta^{\mathrm{J}} \text { passes the position of } d_{k} \\ 1 & \text { otherwise }\end{cases}
$$


Example 6.16. Let us consider the following path $\mathbf{p} \in \mathcal{P}_{7}$ in Example 6.3.

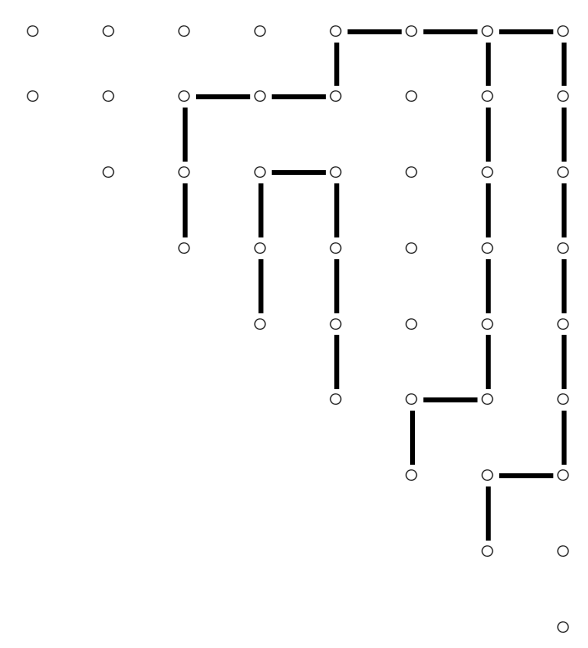

Then $\mathbf{p} \cap \Delta^{\mathrm{J}}$ and $\mathbf{d}(\mathbf{p})$ are given by
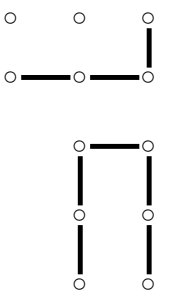

$1 \quad 1$

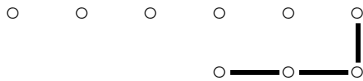

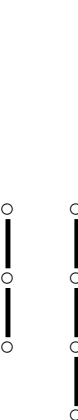

$\left.\right|_{0} ^{0}$

$\begin{array}{lllll}0 & 0 & 1 & 0 & 0\end{array}$

$\begin{array}{lllll}0 & 0 & 1 & 0 & 0\end{array}$

$0 \quad 0$

0

Note that the above array consisting of 0's and 1's corresponds to the sequence

$$
(\mathbf{7}, \mathbf{6}, 5,4,2,3,4, \mathbf{5}, 6,7,1,3, \mathbf{4}, 5,6, \mathbf{2}, 4,5,3,4,2,1, \mathbf{3}, \mathbf{4}, \mathbf{5}, \mathbf{6}, \mathbf{7}) .
$$

Lemma 6.17. The map

$$
\begin{aligned}
\Psi: \mathcal{P}_{n} & \longrightarrow \mathcal{D}^{\mathrm{J}} \\
\mathbf{p} & \longmapsto \mathbf{d}(\mathbf{p})
\end{aligned}
$$

is well-defined and surjective. For $\mathbf{c} \in \mathbf{B}^{\mathrm{J}}$ and $\mathbf{p}, \mathbf{p}^{\prime} \in \Psi^{-1}(\mathbf{d})$, we have

$$
\|\mathbf{c}\|_{\mathbf{p}}=\|\mathbf{c}\|_{\mathbf{p}^{\prime}}
$$

Proof. We state the proof for type $\mathrm{E}_{7}$ with $r=7$. The proof for type $\mathrm{E}_{6}$ is almost identical. Note that the key idea of the proof here is same with [16, Lemma 5.8]. 
Let $\mathbf{p} \in \mathcal{P}_{7}$ be given. To prove $\mathbf{d}(\mathbf{p}) \in \mathcal{D}^{\mathrm{J}}$, we introduce an operator on $\mathcal{P}_{7}$. To do this, let us consider the array with the dashed arrows in (6.5). We denote by $\mathbf{p}^{c}$ the set of dots in $\Delta_{10}$ outside $\mathbf{p}$. For $\mathbf{p}, \mathbf{p}^{\prime} \in \mathcal{P}_{7}$, we define

$$
\mathbf{p} \cong \mathbf{p}^{\prime} \Longleftrightarrow \mathbf{d}(\mathbf{p})=\mathbf{d}\left(\mathbf{p}^{\prime}\right),
$$

and let $\overline{\mathcal{P}_{7}}$ be the set of the equivalent classes of the paths $\mathbf{p} \in \mathcal{P}_{7}$ with respect to the relation $\cong$. Then we define the operator $\mathrm{p}_{i}$ by

$$
\begin{aligned}
\mathrm{p}_{i}: \mathcal{P}_{7} & \longrightarrow \overline{\mathcal{P}_{7}} \cup\{\mathbf{0}\} \\
\mathbf{p} & \longmapsto \mathrm{p}_{i}(\mathbf{p})
\end{aligned}
$$

where $\mathrm{p}_{i}(\mathbf{p})$ is the equivalent class of the paths in $\mathcal{P}_{7}$ determined by changing the dot in $\mathbf{p}^{c} \cap \Delta^{\mathrm{J}}$ located at the position of the leftmost $i$ on the array in (6.5) along the dashed arrow between $i$ 's by one position if it is possible, otherwise we assume $\mathbf{p}_{i}(\mathbf{p})=\mathbf{0}$. For $\mathbf{p}^{\prime} \in \mathbf{p}_{i}(\mathbf{p}) \neq \mathbf{0}$, write $\mathbf{p} \stackrel{\mathbf{p}_{i}}{\longrightarrow} \mathbf{p}^{\prime}$.

For example, if a path $\mathbf{p} \in \mathcal{P}_{7}$ has the black-filled dot outside $\mathbf{p}$ located at the position of 2 in the third row of $(\underline{6.5})$, then $\mathrm{p}_{2}(\mathbf{p}) \neq \mathbf{0}$ and it is a path in $\mathcal{P}_{7}$ determined by interchanging the black-filled dot with the dot located at the position of 2 in the second row of (6.5) along the dashed arrow between 2's.

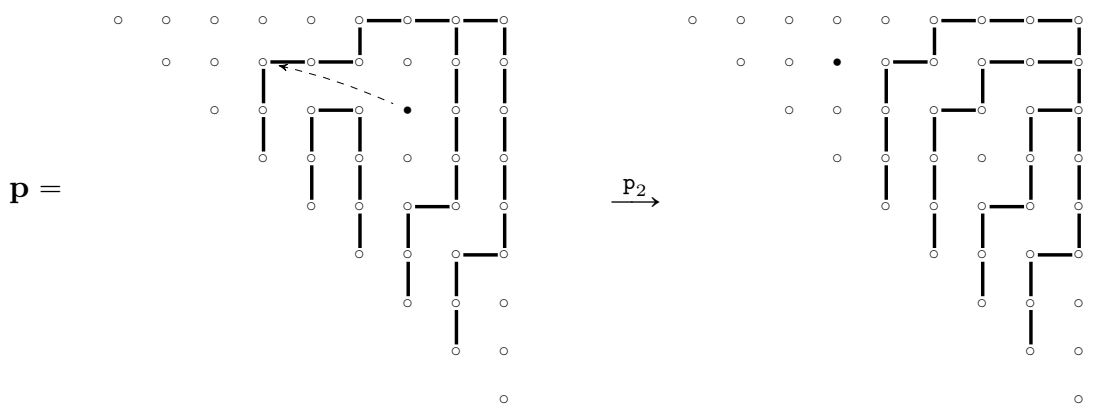

where the path in the right-hand side is a representative in $\mathrm{p}_{2}(\mathbf{p})$.

It is clear that there exists the unique quadruple path $\mathbf{p}_{0}^{\prime}$ in $\mathcal{P}_{7}$ such that $\mathbf{d}\left(\mathbf{p}_{0}^{\prime}\right)=$ $\mathbf{d}\left(\pi_{0}^{\prime}\right)$ (see Remark 6.12). By the configurations of the paths in Definition 6.2(2), one can check that $\mathrm{p}_{i}$ is well-defined and for any $\mathbf{p} \neq \mathbf{p}_{0}^{\prime}$, there exists $i$ such that $\mathbf{p}_{i}(\mathbf{p}) \neq \mathbf{0}$. This implies that $\mathbf{p}_{0}^{\prime}$ is obtained from $\mathbf{p}$ by applying $\mathbf{p}_{i}$ 's successively with finitely many steps, that is, there exists sequences $\left(i_{2}, \ldots, i_{m}\right)$ and $\left(\mathbf{p}_{m}, \ldots, \mathbf{p}_{1}\right)$ such that $\mathbf{p}_{k} \in \mathcal{P}_{7}(1 \leqslant k \leqslant m)$ and

$$
\mathbf{p}=\mathbf{p}_{m} \stackrel{\mathrm{p}_{i_{m}}}{\longrightarrow} \mathbf{p}_{m-1} \stackrel{\mathrm{p}_{i_{m-1}}}{\longrightarrow} \cdots \stackrel{\mathrm{p}_{i_{2}}}{\longrightarrow} \mathbf{p}_{1}=\mathbf{p}_{0}^{\prime} .
$$

Then it allows us to verify that $\mathbf{d}(\mathbf{p})$ is in $\mathcal{D}^{\mathrm{J}}$ for $\mathbf{p} \in \mathcal{P}_{7}$ by an inductive argument on $m$. More precisely, if $\mathbf{d}\left(\mathbf{p}_{m-1}\right) \in \mathcal{D}^{\mathrm{J}}$, then $\mathbf{d}\left(\mathbf{p}_{m-1}\right)$ is one of the $\mathbf{j}_{0}$-trails in Section 6.2.2. Since $\mathbf{p}_{m} \stackrel{\mathbf{p}_{i_{m}}}{\longrightarrow} \mathbf{p}_{m-1}$, the array $\mathbf{d}\left(\mathbf{p}_{m-1}\right)$ is obtained from $\mathbf{d}\left(\mathbf{p}_{m}\right)$ by moving 
1 on $\mathbf{d}\left(\mathbf{p}_{m}\right)$ located at the position of $i_{m}$ along the dashed arrow between $i_{m}$ 's in (6.5). Then it is straightforward to check that if $\mathbf{d}\left(\mathbf{p}_{m}\right) \notin \mathcal{D}^{\mathrm{J}}$, then $\mathbf{p}_{m}$ cannot be a path in $\mathcal{P}_{7}$, which is a contradiction.

By Remark 6.12, for any $\mathbf{d}(\pi)$, the array $\mathbf{d}\left(\pi_{0}^{\prime}\right)=\mathbf{d}\left(\mathbf{p}_{0}^{\prime}\right)$ is obtained from $\mathbf{d}(\pi)$ by moving 1's successively following (6.5) in which each step corresponds to $\mathrm{p}_{i}$ for some $i$. Hence the surjectivity of $\Psi$ is proved.

Finally, for $\mathbf{p} \in \Psi^{-1}(\mathbf{d})$ and $\mathbf{c}=\left(c_{\beta_{k}}\right) \in \mathbf{B}^{\mathrm{J}}$, since $\|\mathbf{c}\|_{\mathbf{p}}$ is equal to the sum of $c_{\beta_{k}}$ 's located at the position of 0's in $\mathbf{d}$, the last statement is proved.

Remark 6.18. We would like to give a technical remark related to paths in type $\mathrm{E}_{6,7}$. Unlike type $\mathrm{E}_{6}$ (cf. Remark 6.5), the subsequence (6.3) of $\mathbf{j}_{0}$-trails $\pi$ at which $\|c\|_{\pi}$ is maximal contains a pair of commuting letters 2 and 3. Due to this difference, the combinatorics of trails for type $\mathrm{E}_{7}$ is more involved (cf. Definition 6.2).

6.2.4. Proof of Theorem 6.4. By Lemma 6.17, there exists a surjection from $\mathcal{P}_{n}$ to $\mathcal{T}^{\prime}$. Then for $\pi \in \mathcal{T}^{\prime}$, we have

$$
\|\mathbf{c}\|_{\pi}=\|\mathbf{c}\|_{\mathbf{p}}
$$

where $\mathbf{p}$ is the corresponding path in $\mathcal{P}_{n}$ under the surjection. Hence, by Lemma 6.10, we obtain the desired formula of $\varepsilon_{r}^{*}(\mathbf{c})$.

\section{REFERENCES}

[1] R. Biswal, T. Scrimshaw, Kirillov-Reshetikhin crystals $B^{7, s}$ for type $E_{7}^{(1)}$, Comm. Algebra. (2021) 1-16.

[2] A. Berenstein, A. Zelevinsky, Tensor product multiplicities, canonical bases and totally positive varieties, Invent. math. 143 (2001) 77-128.

[3] W.H. Burge, Four correspondences between graphs and generalized Young tableaux, J. Combin. Theory Ser. A 17 (1974) 12-30.

[4] N. Bourbaki, Lie groups and Lie algebras, Chapter 4-6, Springer-Verlag Berlin, 2002.

[5] N. Bourbaki, Lie groups and Lie algebras, Chapters 7-9, Springer-Verlag, Berlin, 2005.

[6] V. Chari, On the fermionic formula and the Kirillov-Reshetikhin conjecture, Int. Math. Res. Not. (2001) 629-654.

[7] V. Chari, A. Pressley, Quantum affine algebras and their representations, in: Representations of Groups, in: CMS Conf. Proc., vol. 16, Amer. Math. Soc., Providence, RI, 1995, pp. 59-78.

[8] V. Chari, D. Hernandez, Beyond Kirillov-Reshetikhin modules, Contemp. Math. 506 (2010) 49-81.

[9] G. Fourier, M. Okado, A. Schilling, Kirillov-Reshetikhin crystals for nonexceptional types, Adv. in Math. 222 (2009) 1080-1116.

[10] W. Fulton, Young tableaux, with Application to Representation theory and Geometry, Cambridge Univ. Press, 1997. 
[11] C. Greene, An extension of Schensted's theorem, Adv. Math, 14 (1974) 254-265.

[12] G. Hatayama, A. Kuniba, M. Okado, T. Takagi, Y. Yamada, Remarks on fermionic formula, Contemp. Math. 248 (1999) 243-291.

[13] T. Hiroshima, Perfectness of Kirillov-Reshetikhin crystals $B^{r, s}$ for types $E_{6}^{(1)}$ and $E_{7}^{(1)}$ with a minuscule node $r$, preprint (2021), arXiv:2107.08614.

[14] J. Hong, S.-J. Kang, Introduction to Quantum Groups and Crystal Bases, Graduate Studies in Mathematics 42, Amer. Math. Soc., 2002.

[15] J. E. Humphreys, Reflection Groups and Coxeter Groups, Cambridge University Press, Cambridge, 1990.

[16] I.-S. Jang, J.-H. Kwon, Quantum nilpotent subalgebra of classical quantum groups and affine crystals, J. Combin. Theory Ser. A 168 (2019) 219-254.

[17] I.-S. Jang, J.-H. Kwon, Lusztig data of Kashiwara-Nakashima tableaux in type D, Algebr. Represent. Theor. 24 (2021), no. 4, 959-989.

[18] B. Jones, A. Schilling, Affine structures and a tableau model for $E_{6}$ crystals, J. Algebra 324 (2010) 2512-2542.

[19] V. Kac, Infinite-dimensional Lie algebras, Third edition, Cambridge Univ. Press, 1990.

[20] M. Kashiwara, On crystal bases of the q-analogue of universal enveloping algebras, Duke Math. J. 63 (1991) 465-516.

[21] M. Kashiwara, On crystal bases, Representations of groups, CMS Conf. Proc., vol. 16, Amer. Math. Soc., Providence, RI, (1995) 155-197.

[22] S.-J Kang, M. Kashiwara, K. C. Misra, T. Miwa, T. Nakashima, A. Nakayashiki, Affine crystals and vertex models, In Infinite analysis, Part A, B (Kyoto, 1991), volume 16 of Adv. Ser. Math. Phys., pages 449-484. World Sci. Publ., River Edge, NJ, 1992.

[23] S.-J Kang, M. Kashiwara, K. C. Misra, T. Miwa, T. Nakashima, A. Nakayashiki, Perfect crystals of quantum affine Lie algebras, Duke Math. J. 68 (1992) 499-607.

[24] Y. Kimura, Quantum unipotent subgroup and dual canonical basis, Kyoto J. Math. 52 (2012) 277-331.

[25] D. E. Knuth, Permutations, matrices and generalized Young tableaux, Pacific J. Math. 34 (1970) 709-729.

[26] J.-H. Kwon, RSK correspondence and classically irreducible Kirillov-Reshetikhin crystals, J. Combin. Theory Ser. A 120 (2013) 433-452.

[27] J.-H. Kwon, A crystal embedding into Lusztig data of type A, J. Combin. Theory Ser. A 154 (2018) 422-443.

[28] C. Lenart, S. Naito, D. Sagaki, A. Schilling, M. Shimozono, A uniform model for Kirillov-Reshetikhin crystals II. Alcove model, path model, and $P=X$, Int. Math. Res. Not. IMRN 2017, no. 14, 4259-4319.

[29] C. Lenart, A. Lubovsky, A generalization of the alcove model and its applications, J. Algebraic Combin. 41 (2015), no. 3, 751-783.

[30] C. Lenart, T. Scrimshaw, On higher level Kirillov-Reshetikhin crystals, Demazure crystals, and related uniform models, J. Algebra 539 (2019), 285-304. 
[31] P. Littelmann, Cones, crystals, and patterns, Transform. Groups 3 (1998), no. 2, 145179.

[32] G. Lusztig, Canonical bases arising from quantized enveloping algebras. J. Amer. Math. Soc. 3 (1990) 447-498.

[33] G. Lusztig, Canonical bases arising from quantized enveloping algebras II, Progr. Theoret. Phys. Suppl. 102 (1990) 175-201.

[34] G. Lusztig, Introduction to quantum groups, Progr. Math. Vol. 110, Birkhäuser, 2010.

[35] T. Nakashima, A. Zelevinsky, Polyhedral realizations of crystal bases for quantized Kac-Moody algebras, Adv. Math. 131 (1997), no. 1, 253-278.

[36] K. Naoi, Existence of Kirillov-Reshetikhin crystals of type $G_{2}^{(1)}$ and $D_{4}^{(3)}$, J. Algebra $\mathbf{5 1 2}$ (2018) 47-65.

[37] K. Naoi, T. Scrimshaw, Existence of Kirillov-Reshetikhin crystals for near adjoint nodes in exceptional types, J. Pure Appl. Algebra 225 (2021) no. 5, 106593, 38 pp.

[38] M. Okado, A. Schilling, Existence of Kirillov-Reshetikhin crystals for nonexceptional types, Represent. Theory 12 (2008) 186-207.

[39] Y. Saito, $P B W$ basis of quantized universal enveloping algebras, Publ. Res. Inst. Math. Sci. 30 (1994) 209-232.

[40] B. Salisbury, A. Schultze, P. Tingley, Combinatorial descriptions of the crystal structure on certain PBW bases, Transform. Groups 23 (2018) 501-525.

[41] M. Shimozono, Affine type A crystal structure on tensor products of rectangles, Demazure characters, and nilpotent varieties, J. Algebraic Combin. 15 (2002) 151-187.

[42] J. Stembridge, On the fully commutative elements of Coxeter groups, J. Algebraic Combin. 5 (1996), no. 4, 353-385.

[43] P. Papi, A characterization of a special ordering in a root system, Proc. Amer. Math. Soc. 120 (1994) 661-665.

Department of Mathematical Sciences, Seoul National University, Seoul 08826, REPUBLIC OF KOREA

Email address: is_jang@snu.ac.kr 